J. DIFFERENTIAL GEOMETRY

55 (2000) 145-191

\title{
ANALYSIS ON GROUPS OF DIFFEOMORPHISMS OF MANIFOLDS WITH BOUNDARY AND THE AVERAGED MOTION OF A FLUID
}

\author{
STEVE SHKOLLER
}

\begin{abstract}
We establish the existence of three new subgroups of the group of volumepreserving diffeomorphisms of a compact $n$-dimensional $(n \geq 2)$ Riemannian manifold which are associated with the Dirichlet, Neumann, and Mixed type boundary conditions that arise in second-order elliptic PDEs. We prove that when endowed with the $H^{s}$ Hilbert-class topologies for $s>(n / 2)+$ 1 , these subgroups are $C^{\infty}$ differential manifolds. We consider these new diffeomorphism groups with an $H^{1}$-equivalent right invariant metric, and prove the existence of unique smooth geodesics $\eta(t, \cdot)$ of this metric, as well as existence and uniqueness of the Jacobi equations associated to this metric. Geodesics on these subgroups are, in fact, the flows of a timedependent velocity vector field $u(t, x)$, so that $\partial_{t} \eta(t, \cdot)=u(t, \eta(t, \cdot))$ with $\eta(0, x)=x$, and remarkably the vector field $u(t, x)$ solves the so-called Lagrangian averaged Euler (LAE- $\alpha$ ) equations on $M$. These equations, and their viscous counterparts, the Lagrangian averaged Navier-Stokes (LANS$\alpha$ ) equations, model the motion of a fluid at scales larger than an a priori fixed parameter $\alpha>0$, while averaging (or filtering-out) the small scale motion, and this is achieved without the use of artificial viscosity. We prove that for divergence-free initial data satisfying $u=0$ on $\partial M$, the LAE- $\alpha$ equations are well-posed, globally when $n=2$. We also find the boundary conditions that make the LANS- $\alpha$ equations well-posed, globally when $n=3$, and prove that solutions of the LANS- $\alpha$ equations converge when $n=2,3$ for almost all $t$ in some fixed time interval $(0, T)$ in $H^{s}$, $s \in(n / 2+1,3)$ to solutions of the LAE- $\alpha$ equations, thus confirming the scaling arguments of Barenblatt \& Chorin.
\end{abstract}

Received April 26, 2000.

1991 Mathematics Subject Classification. 35Q35, 35Q53, 58B20, 58D05. 


\section{Introduction}

The Lagrangian averaged Euler (LAE- $\alpha$ ), or Euler- $\alpha$, equations may be written in Euclidean space $\mathbb{R}^{n}$ as the following system of partial differential equations (PDE):

$$
\begin{gathered}
\partial_{t}\left(1-\alpha^{2} \triangle\right) u+\operatorname{curl}\left(1-\alpha^{2} \triangle\right) u \times u=-\operatorname{grad} p, \\
u(0)=u_{0}, \quad \operatorname{div} u=0,
\end{gathered}
$$

where $u(t, x)$ is the velocity vector field, $p(t, x)$ is the scalar pressure, and $\alpha>0$ is the averaging length-scale. This conservative system of PDE models the averaged motion of an ideal incompressible fluid, filtering over spatial scales smaller than $\alpha$ (see [20] and [24] for derivations of this model). Let $P$ denote the Hodge (or Leray) projector defined below in Section 5, and let $A=-P \triangle$ denote the Stokes operator. When dissipation is present due to kinematic viscosity $\nu>0$, we obtain the so-called the Lagrangian averaged Navier-Stokes (LANS- $\alpha$ ), or NavierStokes- $\alpha$, equations:

$$
\begin{gathered}
\partial_{t}\left(1-\alpha^{2} \triangle\right) u+\operatorname{curl}\left(1-\alpha^{2} \triangle\right) u \times u=-\operatorname{grad} p-\nu\left(1-\alpha^{2} \triangle\right) A u, \\
u(0)=u_{0}, \quad \operatorname{div} u=0 .
\end{gathered}
$$

The LANS- $\alpha$ equations were first studied in [17] with periodic boundary conditions; the extension to bounded domains will be addressed herein and in [24]. We remark that when $\alpha=0$ the Euler $(\nu=0)$ and NavierStokes $(\nu>0)$ equations are recovered. Equations (1.1) and (1.2) have received a great deal of attention in both the mathematics and physics literature, because of their remarkable ability to reproduce the largescale averaged motion of the Euler and Navier-Stokes equations, without the use of artificial viscosity or dissipation. We refer the interested reader to the papers [7], [8], [9], [27], [10], [30], and [29] for numerical simulations of turbulent flows using the LANS- $\alpha$ equations, as well as (energy) scaling arguments, and modifications of the morphology of fluid structure.

In a striking coincidence, if the damping term $\nu \triangle u$ is added to the right-hand-side of (1.1) instead of the dissipative term $-\nu\left(1-\alpha^{2} \triangle\right) A u$, then the equations are precisely the Rivlin-Ericksen second-grade fluids equations developed in [33]; furthermore, (1.1) can be exactly identified with vortex blob methods (introduced in [11]), and in two-dimensions are globally well-posed for Radon measure initial data [30]. 
Thus far, there has been no analytic study of any kind for either the Lagrangian averaged Euler (LAE- $\alpha$ ) or Lagrangian averaged NavierStokes (LANS- $\alpha$ ) equations on Riemannian manifolds with boundary. In this paper, we shall generalize (1.1) and (1.2) to the setting of a compact, $n$-dimensional $(n \geq 2)$, Riemannian manifold with smooth boundary. In the inviscid case, we shall extend our results from [34] and [23], and prove that solutions of the LAE- $\alpha$ equations are geodesics on new subgroups of the volume-preserving diffeomorphism group with respect to a very natural (new) $H^{1}$-equivalent right-invariant weak metric. Because of this fact, there is a wonderful interplay between the geometry and curvature of the new volume-preserving diffeomorphism groups which we introduce, and the analytical properties of solutions to both the LAE- $\alpha$ and LANS- $\alpha$ equations. In the geometric setting, one can view this work as an extension of the program initiated by Arnold [3], [4] and Ebin \& Marsden [13] to the setting of turbulence modeling. In the PDE setting, our intent is to write (1.1) in Lagrangian variables, and study the resulting Lagrangian PDE; as we shall explain below, there is greater temporal regularity available when studying the Lagrangian PDE rather than the Eulerian PDE, as well as interesting geometric implications to the study of volume-preserving diffeomorphisms.

We shall establish $C^{\infty}$-in-time local well-posedness in any dimension for the LAE- $\alpha$ equations with $H^{s}$ initial data, $s>(n / 2)+1$, by proving that unique $C^{\infty}$ geodesics exist; solutions are global in dimension two. These results are analogous to the fundamental results of [3] and [13], wherein it was proved that solutions of the incompressible Euler equations are smooth geodesics of the weak $L^{2}$ right-invariant metric. In our setting, however, we must first establish the existence of new $C^{\infty}$ volume-preserving diffeomorphism groups on manifolds with boundary, such that motion on these subgroups corresponds to solutions of both the LAE- $\alpha$ and LANS- $\alpha$ equations. We remark that it is quite easy to establish a local existence theory in Eulerian variables, and we do this in the proof of Theorem 3.

In the viscous case, we shall derive the boundary conditions which provide well-posedness for the LANS- $\alpha$ equations when boundaries are present. Unlike the three-dimensional Navier-Stokes equations, the threedimensional LANS- $\alpha$ equations are globally well-posed (see [17] for spatially periodic solutions, and [25] for solutions on bounded domains). We establish that solutions of the LANS- $\alpha$ equations converge regularly to solutions of the LAE- $\alpha$ equations even in the presence of boundaries. This result verifies the scaling arguments used by Barenblatt \& Chorin 
in $[5]$.

\section{A generalization to Riemannian manifolds}

We begin by generalizing the system of equations (1.1) from $\mathbb{R}^{n}$ to the setting of a $C^{\infty}$, compact, oriented, $n$-dimensional Riemannian manifold with $C^{\infty}$ boundary, $(M, g)$. Letting $\nabla$ denote the Levi-Civita covariant derivative, (1.1) becomes

$$
\begin{aligned}
& \begin{aligned}
& \partial_{t}\left(1-\alpha^{2} \triangle_{r}\right) u-\nu \triangle_{r} u+\nabla_{u}\left(1-\alpha^{2} \triangle_{r}\right) u \\
&-\alpha^{2}(\nabla u)^{t} \cdot \triangle_{r} u=-\operatorname{grad} p, \\
& \operatorname{div} u=0, \quad u(0)= u_{0},
\end{aligned}
\end{aligned}
$$

where $\alpha>0$ is a constant and

$$
\triangle_{r}=-(d \delta+\delta d)+2 \text { Ric. }
$$

We supplement this PDE with one of the following three boundary conditions:

(a) Dirichlet or no-slip: $u=0$ on $\partial M$.

(b) Neumann or free-slip: $g(u, n)=0$ and $\left(\nabla_{n} u\right)^{\tan }+S_{n}(u)=0$ on $\partial M$.

(c) Mixed: $u=0$ on $\Gamma_{1}$, and $g(u, n)=0,\left(\nabla_{n} u\right)^{\tan }+S_{n}(u)=0$ on $\Gamma_{2}$, where $\partial M=\Gamma_{1} \cup \Gamma_{2}, \bar{\Gamma}_{1}=\partial M / \Gamma_{2}$, and the sets $\Gamma_{1}, \Gamma_{2}$ are disjoint.

On a Riemannian manifold, there is always more than one choice for the correct "Laplacian" on vector fields or 1-forms. Our Laplacian $\triangle_{r}$ is the operator

$$
\mathcal{L}=-2 \text { Def }^{*} \text { Def }
$$

acting on divergence-free vector fields (or coexact 1-forms), where the (rate of) deformation tensor is given by

$$
\operatorname{Def}(u)=\frac{1}{2}\left(\nabla u+\nabla u^{t}\right)=\frac{1}{2} £_{u} g, \quad £=\text { Lie derivative, }
$$

and Def* is the $L^{2}$ formal adjoint of Def. Other possible choices are the Hodge Laplacian $-\triangle=(d \delta+\delta d)$ or the rough Laplacian $-\operatorname{Tr} \nabla \nabla$, but the boundary conditions (a)-(c) insist upon our choice $\mathcal{L}$. Note that

$$
\left(\nabla_{n} u\right)^{\tan }+S_{n}(u)=[\operatorname{Def}(u) \cdot n]^{\tan }
$$


for vector fields $u$ which are tangential to $\partial M$, so the second-fundamental form of the boundary $\partial M$ is encoded into the natural boundary condition; this is a significant improvement over the energy function used in [23], and shows that the curvature of the boundary contributes to the energy of the averaged fluid motion.

\subsection{Reformulation of the LAE- $\alpha$ and LANS- $\alpha$ equations}

All of our analytical results are founded on a reformulation of the LAE$\alpha$ and LANS- $\alpha$ equations. We shall show that the LAE- $\alpha$ equations have the equivalent form:

$$
\begin{gathered}
\partial_{t} u+\nabla_{u} u+\mathcal{U}^{\alpha}(u)+\mathcal{R}^{\alpha}(u)=-\left(1-\alpha^{2} \mathcal{L}\right)^{-1} \operatorname{grad} p \\
\operatorname{div} u(t, x)=0, \\
u=0 \text { on } \partial \mathrm{M} \\
u(0, x)=u_{0}(x)
\end{gathered}
$$

where

$$
\mathcal{U}^{\alpha}(u)=\alpha^{2}\left(1-\alpha^{2} \mathcal{L}\right)^{-1} \operatorname{Div}\left[\nabla u \cdot \nabla u^{t}+\nabla u \cdot \nabla u-\nabla u^{t} \cdot \nabla u\right],
$$

and

$$
\mathcal{R}^{\alpha}(u)=\alpha^{2}\left(1-\alpha^{2} \mathcal{L}\right)^{-1}\{\operatorname{Tr}[\nabla(R(u, \cdot) u)+R(u, \cdot) \nabla u+R(\nabla u, \cdot) u]
$$

$$
\left.-\left(\nabla_{u} \operatorname{Ric}\right) \cdot u+\nabla u^{t} \cdot \operatorname{Ric}(u)\right\}
$$

and that the LANS- $\alpha$ equations are given by

$$
\partial_{t} u+\nabla_{u} u+\mathcal{U}^{\alpha}(u)+\mathcal{R}^{\alpha}(u)=-\left(1-\alpha^{2} \mathcal{L}\right)^{-1} \operatorname{grad} p-\nu A u,
$$

together with (1.4c) and (1.4d), where

$$
A=-P \triangle_{r}
$$

denotes the Stokes operator on Riemannian manifolds. The regularizing terms $\mathcal{U}^{\alpha}(u)$ and $\mathcal{R}^{\alpha}(u)$ are not dissipative; rather, they remove energy from the scales smaller than $\alpha$ in a dispersive fashion.

In the simplest setting of the flat torus $\mathbb{T}^{n}$ (periodic box) with $R=0$, the LANS- $\alpha$ equations become

$$
\begin{gathered}
\partial_{t} u+\nabla_{u} u+\operatorname{Div} \tau^{\alpha}=-\operatorname{grad} p+\nu \Delta u \\
\tau^{\alpha}(u)=\alpha^{2}\left(1-\alpha^{2} \mathcal{L}\right)^{-1}\left[\nabla u \cdot \nabla u^{t}+\nabla u \cdot \nabla u-\nabla u^{t} \cdot \nabla u\right] .
\end{gathered}
$$


The term $\tau^{\alpha}$ may be thought of a subgrid stress analogous to the traditional Reynolds stress, but unlike Reynolds Averaged Navier-Stokes (RANS) or Large Eddy Simulation (LES) models, the additional term Div $\tau^{\alpha}$ is not dissipative. In particular, no artificial dissipation is added to the Navier-Stokes equations to filter the small scale fluid motion; rather, a geometric, nonlinear-dispersion-type mechanism is used to averaged over spatial scales smaller than $\alpha$. The idea of smoothing by nonlinear dispersion was used extensively by Holm, Ratiu, \& Marsden [19].

The form of LANS- $\alpha$ given by (1.7) also solves the problem of boundary conditions; namely, it is clear that for all solutions to LANS- $\alpha, A u$ must equal zero on the boundary, since all of the other terms in the equation equal zero on the boundary, whenever $u=0$ on $\partial M$. It thus makes sense to write the LANS- $\alpha$ equations as

$$
\begin{aligned}
\partial_{t}\left(1-\alpha^{2} \triangle_{r}\right) u+\nabla_{u}\left(1-\alpha^{2} \triangle_{r}\right) u-\alpha^{2}(\nabla u)^{t} \cdot \triangle_{r} u \\
=-\operatorname{grad} p-\nu\left(1-\alpha^{2} \triangle_{r}\right) A u \\
u=0 \text { and } \triangle_{r} u=0 \text { on } \partial M,
\end{aligned}
$$

since for sufficiently regular velocity fields, $A u$ is in the domain $D(1-$ $\left.\alpha^{2} \triangle_{r}\right)=H^{2}(T M) \cap H_{0}^{1}(T M)$. Both forms of the LANS- $\alpha$ equations, (1.7) and (1.9), are equivalent, but it is (1.7) which provides the additional boundary condition to deal with the fourth-order operator $\nu(1-$ $\left.\alpha^{2} \triangle_{r}\right) A$ on bounded domains.

\subsection{Notation}

For each $x \in \partial M$, the $g$-orthogonal bundle splitting $T_{x} M=T_{x} \partial M \oplus N_{x}$ induces the Whitney sum

$$
\left.T M\right|_{\partial M}=T \partial M \oplus_{g} N
$$

where $N$ is the normal bundle, $N=\cup_{x \in \partial M} N_{x} \downarrow \partial M$.

Letting $\pi: E \rightarrow M$ be a vector bundle over $M$ (or over $\partial M$ ), we denote the $H^{s}$ sections of $E$ by $H^{s}(E)$ and for all $\eta \in \mathcal{D}^{s}$, we set $H_{\eta}^{s}(E):=\left\{U \in H^{s}(M, E) \mid \pi \circ U=\eta\right\}$.

For any vector bundle $\mathcal{E}$ over a base manifold $\mathcal{M}$, we shall often make use of the notation $\mathcal{E}_{m} \downarrow \mathcal{M}$ to denote $\mathcal{E}$, where $\mathcal{E}_{m}$ is the fiber over $m \in \mathcal{M}$. 
We use $R$ to denote the Riemannian curvature operator of $\nabla$. The Ricci curvature as a bilinear form is given by

$$
\operatorname{Ric}(x, y)=\operatorname{Tr} g(R(x, \cdot) \cdot, y),
$$

with the associated linear operator Ric: $T_{x} M \rightarrow T_{x} M$ given by

$$
g(\operatorname{Ric}(x), y)=\operatorname{Ric}(x, y) \text {. }
$$

If $\eta \in H^{s}(M, M)$, then the tangent mapping $T \eta$ is in

$$
H^{s-1}\left(M, T^{*} M \otimes \eta^{*}(T M)\right) .
$$

If $w \in T_{x} M$, then in a local chart,

$$
T \eta(x) \cdot w=(\eta(x), D \eta(x) \cdot w)
$$

where $D$ is the matrix of partial derivatives of $\eta$ with respect to the coordinate chart.

We shall use the symbol $£$ to denote the Lie derivative, $d$ for the exterior derivative on $\Lambda^{k}(M)$, the differential $k$-forms on $M$, and $\delta$ for its formal adjoint with respect to the $L^{2}$ pairing. For a vector field $u$ on $M, \nabla u^{t}$ shall denote the transpose of $\nabla u$ with respect to $g$.

The Hodge Laplacian on differential $k$-forms is $\triangle=-(d \delta+\delta d)$, and

$$
\triangle_{r}=\triangle+2 \text { Ric. }
$$

When we wish to explicitly convert between vector fields and 1forms, we shall use the musical maps $b: T M \rightarrow T^{*} M$ and $\sharp: T^{*} M \rightarrow$ $T M$; for example, if $u$ is a vector field on $M$, then $u^{b}$ is the associated 1 -form.

\section{Main results}

We prove the existence of smooth-in-time classical solutions to the Lagrangian form of (1.3); namely, we study the Cauchy problem for the Lagrangian flow map on any one of three subgroups (which we construct) of $\mathcal{D}_{\mu}^{s}$. We let $\mathcal{D}_{\mu}^{s}$ denote the topological group consisting of Hilbert $H^{s}$-class volume-preserving diffeomorphism of $M$ with $H^{s}$ inverses. Solutions of the LAE- $\alpha$ equations have better temporal regularity in Lagrangian variables than in Eulerian variables and contain the Riemannian geometry of the volume-preserving diffeomorphism group as we shall describe below. 


\section{New subgroups of $\mathcal{D}_{\mu}^{s}$}

For $\eta \in \mathcal{D}_{\mu}^{s}, s>\frac{n}{2}+1$, we let $T \eta$ denote its tangent map, i.e., the Frechet derivative of $\eta$ thought of as bundle map. In local coordinate $x^{i}, T \eta(x)$ is $\partial \eta^{i} / \partial x^{j}(x)$.

Theorem 1. Set $s>\frac{n}{2}+1$ and let $n$ denote the outward-pointing normal field along the boundary $\partial M$, and let $S_{n}: T \partial M \rightarrow T \partial M$ denote the Weingarten map so that

$$
g\left(S_{n}(u), v\right)=I I_{n}(u, v)=-g\left(\nabla_{u} n, v\right), \quad u, v \in H^{s-\frac{3}{2}}(T \partial M),
$$

where $I I_{n}$ is the second fundamental form of $\partial M \subset M$. Define the sets

$$
\begin{aligned}
& \mathcal{D}_{\mu, N}^{s}=\left\{\eta \in \mathcal{D}_{\mu}^{s}|T \eta|_{\partial M} \cdot n \in H_{\eta}^{s-3 / 2}(N), \text { for all } n \in H^{s-1 / 2}(N)\right\}, \\
& \mathcal{D}_{\mu, D}^{s}=\left\{\eta \in \mathcal{D}_{\mu}^{s}|\eta|_{\partial M}=e\right\},
\end{aligned}
$$

and

$$
\begin{aligned}
& \mathcal{D}_{\mu, m i x}^{s}=\left\{\eta \in \mathcal{D}_{\mu}^{s} \mid \eta \text { leaves } \Gamma_{i} \text { invariant, }\left.\quad \eta\right|_{\Gamma_{1}}=e,\right. \\
&\left.\left.T \eta\right|_{\Gamma_{2}} \cdot n \in H^{s-3 / 2}\left(N \mid \Gamma_{2}\right), \text { for all } n \in H^{s-1 / 2}\left(N \mid \Gamma_{2}\right)\right\},
\end{aligned}
$$

where we suppose that $M, \partial M$ are $C^{\infty}$, that $\Gamma_{1}$ and $\Gamma_{2}$ are two disjoint subsets of $\partial M$ such that if $m_{0} \in \Gamma_{i}(i=1,2)$, a local chart $U$ (in $\bar{M}$ ) about $m_{0}$ can be chosen so that $\bar{U} \cap \partial M \subset \Gamma_{i}$; furthermore, we assume that $\bar{\Gamma}_{1}=\partial M / \Gamma_{2}$ and that $\partial M=\Gamma_{1} \cup \Gamma_{2}$.

Then $\mathcal{D}_{\mu, D}^{s}, \mathcal{D}_{\mu, N}^{s}$, and $\mathcal{D}_{\mu, \text { mix }}^{s}$ are all $C^{\infty}$ subgroups of $\mathcal{D}_{\mu}^{s}$, and the tangent space at the identity of these groups is given by

$$
\begin{aligned}
T_{e} \mathcal{D}_{\mu, N}^{s}=\left\{u \in T_{e} \mathcal{D}_{\mu}^{s} \mid\left(\left.\nabla_{n} u\right|_{\partial M}\right)^{\tan }+S_{n}(u)=0 \in H^{s-3 / 2}(T \partial M)\right. \\
\quad \\
\left.\quad \text { for all } n \in H^{s-1 / 2}(N)\right\}, \\
T_{e} \mathcal{D}_{\mu, D}^{S}=\left\{u \in T_{e} \mathcal{D}_{\mu}^{s}|u|_{\partial M}=0\right\},
\end{aligned}
$$

and

$$
\begin{gathered}
T_{e} \mathcal{D}_{\mu, \text { mix }}^{S}=\left\{u \in T_{e} \mathcal{D}_{\mu}^{s} \mid\left(\left.\nabla_{n} u\right|_{\partial M}\right)^{\tan }+S_{n}(u)=0 \in H^{s-\frac{3}{2}}\left(T \Gamma_{2}\right)\right. \\
\text { for all } \left.n \in H^{s-\frac{1}{2}}\left(N \mid \Gamma_{2}\right) \text { and }\left.u\right|_{\Gamma_{1}}=0\right\} .
\end{gathered}
$$

We also form the corresponding sets $\mathcal{D}_{N}^{s}, \mathcal{D}_{D}^{s}$, and $\mathcal{D}_{\text {mix }}^{s}$ which do not have the volume-preserving constraint imposed. These sets are $C^{\infty}$ subgroups of the full diffeomorphism group $\mathcal{D}^{s}$, and have the analogous tangent spaces at the identity without the divergence-free constraint.

We call the groups $\mathcal{D}_{\mu, D}^{s}, \mathcal{D}_{\mu, N}^{s}$, and $\mathcal{D}_{\mu, m i x}^{s}$, the Dirichlet, Neumann, and mixed volume-preserving diffeomorphism groups. 


\section{Well-posedness in Lagrangian variables}

Theorem 1 allows us to do smooth calculus on these spaces, and hence consider the Lagrangian flow of (1.3). In this article, we shall prove results for the case of the group $\mathcal{D}_{\mu, D}^{s}$, as the no-slip conditions have been of most interest in the literature. ${ }^{1}$

Theorem 2. Set $s>(n / 2)+1$, and let $\langle\cdot, \cdot\rangle$ denote the $H^{1}$ equivalent right-invariant metric on $\mathcal{D}_{\mu, D}^{s}$ given at the identity by

$$
\langle X, Y\rangle_{e}=(X, Y)_{L^{2}}+2 \alpha^{2}(\operatorname{Def}(X), \operatorname{Def}(Y))_{L^{2}}, \quad \forall X, Y \in T_{e} \mathcal{D}_{\mu, D}^{s} .
$$

For $u_{0} \in T_{e} \mathcal{D}_{\mu, D}^{s}$, there exists an interval $I=(-T, T)$, depending on $\left|u_{0}\right|_{s}$, and a unique geodesic $\dot{\eta}$ of $\langle\cdot, \cdot\rangle$ with initial data $\eta(0)=e$ and $\dot{\eta}(0)=u_{0}$ such that $\dot{\eta}$ is in $C^{\infty}\left(I, T \mathcal{D}_{\mu, D}^{s}\right)$ and has $C^{\infty}$ dependence on the initial velocity $u_{0}$.

The geodesic $\eta$ is the Lagrangian flow of the divergence-free timedependent vector field $u(t, x)$ given by

$$
\partial_{t} \eta(t, x)=u(t, \eta(t, x)), \quad \eta(0)=e,
$$

and $u \in C^{0}\left(I, T_{e} \mathcal{D}_{\mu, D}^{s}\right) \cap C^{1}\left(I, T_{e} \mathcal{D}_{\mu, D}^{s-1}\right)$ uniquely solves (1.3), and depends continuously on $u_{0}$.

We use $\dot{\eta}(t, x)$ to denote $\partial_{t} \eta(t, x)$.

Corollary 1. If $n=\operatorname{dim}(M)=2$, then $T=\infty$, so $C^{\infty}$ geodesics of $\langle\cdot, \cdot\rangle$ on $\mathcal{D}_{\mu, D}^{s}$ exist for all time.

The proof, which is based on the fact that $\left|\operatorname{curl}\left(1-\alpha^{2} \triangle_{r}\right) u\right|_{L^{2}}$ is conserved, is given by Theorem 2.1 in [35], where the same result is also shown to hold for the Euler equations.

\section{Limit of zero viscosity}

In certain topologies which are not too strong, we can also prove the limit of zero viscosity; namely, solutions of the LANS- $\alpha$ equations converge to solutions of the LAE- $\alpha$ equations on bounded domains, with the no-slip $u=0$ boundary condition.

Theorem 3 For $n=2,3$ and $s \in\left(\frac{n}{2}+1,3\right)$ solutions of the LANS$\alpha$ equations (1.7) converge in $L^{\infty}\left((0, T), T_{e} \mathcal{D}_{\mu, D}^{s}\right)$ to solutions of the LAE- $\alpha$ equations (1.4).

\footnotetext{
${ }^{1}$ By setting $\partial M=\emptyset$, all of our results hold for compact oriented $n$ dimensional Riemannian manifolds without boundary.
} 
This theorem confirms the scaling arguments of Barenblatt-Chorin (see, for example, the second paragraph of [5]). We remark that when $s \geq 3$, the LANS- $\alpha$ equations satisfy the additional boundary condition $A u=0$ which the LAE- $\alpha$ do not; thus, in such strong topologies, a boundary layer forms, and requires the development of a Prandlt-type theory.

\section{Riemannian geometry of $\left(\mathcal{D}_{\mu, D}^{s},\langle\cdot, \cdot\rangle\right)$}

There are some interesting geometric corollaries which Theorem 2 provides. We define the Riemannian exponential map $\mathcal{E x p}_{e}: T_{e} \mathcal{D}_{\mu, D}^{s} \rightarrow$ $\mathcal{D}_{\mu, D}^{s}$ of the right invariant metric $\langle\cdot, \cdot\rangle$ by $\mathcal{E}_{\mathrm{xp}_{e}}(t u)=\eta(t)$, where $t>0$ is sufficiently small, and $\eta(t)$ is the geodesic curve on $\mathcal{D}_{\mu, D}^{s}$ emanating from $e$ with initial velocity $u$. Because the above theorem guarantees that geodesics of $\langle\cdot, \cdot\rangle$ have $C^{\infty}$ dependence on initial data, $\mathcal{E x p}_{e}$ is well defined, satisfies $\mathcal{E x p}_{e}(0)=e$, and so by the inverse function theorem we obtain

Corollary 2. For $s>(n / 2)+1$, the Riemannian exponential map

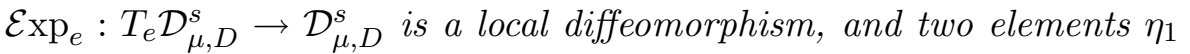
and $\eta_{2}$ of $\mathcal{D}_{\mu, D}^{s}$ that are in a sufficiently small neighborhood of e can be connected by a unique geodesic of $\langle\cdot, \cdot\rangle$ in $\mathcal{D}_{\mu, D}^{s}$.

Note that for the $L^{2}$ right invariant metric on $\mathcal{D}_{\mu}^{s}$ whose geodesic flow gives solutions to the Euler equations, the analogous local result was obtained by Ebin-Marsden [13], but Shnirelman [36] has shown that this local result does not hold globally. Namely, when $M$ is the unit cube in $\mathbb{R}^{3}$, he proved the existence of fluid configurations which cannot be connected to the identity by an energy minimizing curve. This has motivated the construction of generalized flows; Brenier [6] has recently constructed Young measure-valued flows that are both Lagrangian and Eulerian in character, and which give weak solutions to the Euler equations in the sense of connecting any two fluid configurations (again on the unit cube in $\mathbb{R}^{3}$ ). The construction of such weak solutions for the weak form of (1.3), given on the flat three-torus $\mathbb{T}^{3}$ by

$$
\begin{aligned}
\int_{0}^{T} \int_{\mathbb{T}^{3}}\left\{-u \cdot \partial_{t} \phi-u \otimes u: \nabla \phi\right. \\
\left.\quad+\alpha^{2}\left(1-\alpha^{2} \triangle\right)^{-1}\left[\nabla u \cdot \nabla u^{t}+\nabla u \cdot \nabla u-\nabla u^{t} \cdot \nabla u\right]: \nabla \phi\right\} d x d t=0
\end{aligned}
$$

for all $\phi \in C^{\infty}\left([0, T] \times \mathbb{T}^{3}\right)$ with $\operatorname{div} \phi=0$, is the subject of ongoing research. In this setting, one generates weak solutions whose distributional derivatives are Young measures. 
Note that while the group exponential map is only $C^{0}$ and does not cover a neighborhood of the identity, the Riemannian exponential map on $\mathcal{D}_{\mu, D}^{s}$ is smooth by Theorem 2, so that in conjunction with the fact that the right multiplication map is $C^{\infty}$, the topological group $\mathcal{D}_{\mu, D}^{s}$ looks very much like a Lie group. As a consequence of the smoothness of $\mathcal{E} \mathrm{xp}_{e}$ and the proof of Theorem 12.1 in [13], geodesics of $\langle\cdot, \cdot\rangle$, which are the solutions of (1.3), instantly inherit the regularity of the initial data. Thus,

Corollary 3. For $s>\frac{n}{2}+1$, let $\eta(t)$ be a geodesic of the right invariant metric $\langle\cdot, \cdot\rangle$ on $\mathcal{D}_{\mu, D}^{s}$, i.e., $\partial_{t} \eta(t, x)=u(t, \eta(t, x))$ and $u(t, x)$ is the unique solution of (1.3) with $\nu=0$. If $\eta(0) \in \mathcal{D}_{\mu, D}^{s+k}$ and $\dot{\eta}(0) \in$ $T_{\eta(0)} \mathcal{D}_{\mu, D}^{s+k}$ for $0 \leq k \leq \infty$, then $\eta(t)$ is $H^{s+k}$ for all $t \in I$.

Our final theorem is geometric and concerns the existence of the weak Levi-Civita covariant derivative on $\mathcal{D}_{\mu, D}^{s}$ of the the weak right invariant metric $\langle\cdot, \cdot\rangle$, as well as its Riemannian curvature operator.

Because the metric $\langle\cdot, \cdot\rangle$ is equivalent to an $H^{1}$ metric by Korn's inequality, it induces a weak topology relative to the strong $H^{s}$ topology, $s>\frac{n}{2}+1$, of $\mathcal{D}_{\mu, D}^{s}$. In general, there does not exist a weak covariant derivative operator associated to a weak metric, nor a bounded Riemannian curvature operator. Thanks to Theorem 2, however, these structures do indeed exist.

Theorem 4. Extending $X_{\eta}, Y_{\eta}, Z_{\eta} \in T_{\eta} \mathcal{D}_{\mu, D}^{s}, \eta \in \mathcal{D}_{\mu, D}^{s}$, to smooth vector fields $X, Y, Z$ on $\mathcal{D}_{\mu, D}^{s}$, there exists a right invariant unique LeviCivita covariant derivative $\tilde{\nabla}$ of $\langle\cdot, \cdot\rangle$ on $\mathcal{D}_{\mu, D}^{s}$ given by

$$
\begin{aligned}
\tilde{\nabla}_{X} Y(\eta)= & \left\{\mathcal { P } _ { e } \circ \left[\partial_{t}\left(Y_{\eta} \circ \eta^{-1}\right)+\nabla_{X_{\eta} \circ \eta^{-1}}\left(Y_{\eta} \circ \eta^{-1}\right)\right.\right. \\
& \left.\left.+\frac{1}{2}\left(\mathfrak{U}\left(X_{\eta} \circ \eta^{-1}, Y_{\eta} \circ \eta^{-1}\right)+\mathfrak{R}\left(X_{\eta} \circ \eta^{-1}, Y_{\eta} \circ \eta^{-1}\right)\right)\right]\right\} \circ \eta,
\end{aligned}
$$

where $\mathfrak{U}$ and $\mathfrak{R}$ are given by polarization of the operators $\mathcal{U}^{1}$ and $\mathcal{R}^{1}$, defined in (1.5) and (1.6), respectively, and where for $r \geq 1$,

$$
\mathcal{P}_{e}: H^{r}(T M) \cap H_{0}^{1}(T M) \rightarrow V_{\mu}^{r}
$$

is the $\langle\cdot, \cdot\rangle_{e}$-orthogonal projection given by

$$
\mathcal{P}_{e}(F)=v,
$$

where $v \in \mathcal{V}_{\mu}^{r}$ is the unique solution of the Stokes problem

$$
\begin{gathered}
(1-\mathcal{L}) v+\operatorname{grad} p=(1-\mathcal{L}) F \\
\operatorname{div} v=0, \quad v=0 \text { on } \partial M
\end{gathered}
$$


For right-invariant vector fields $X, Y$ on $\mathcal{D}_{\mu, D}^{s}$ which are completely determined by there value at the identity $X_{e}, Y_{e}$,

$$
\tilde{\nabla}_{X} Y(e)=\mathcal{P}_{e} \circ\left[\nabla_{X_{e}} Y_{e}+\frac{1}{2}\left(\mathfrak{U}\left(X_{e}, Y_{e}\right)+\mathfrak{R}\left(X_{e}, Y_{e}\right)\right)\right] .
$$

Finally, define the weak Riemannian curvature tensor

$$
\tilde{R}_{\eta}:\left[T_{\eta} \mathcal{D}_{\mu, D}^{s}\right]^{3} \rightarrow T_{\eta} \mathcal{D}_{\mu, D}^{s}
$$

by

$\tilde{R}_{\eta}\left(X_{\eta}, Y_{\eta}\right) Z_{\eta}=\left(\tilde{\nabla}_{Y} \tilde{\nabla}_{X} Z\right)_{\eta}-\left(\tilde{\nabla}_{X} \tilde{\nabla}_{Y} Z\right)_{\eta}+\left(\tilde{\nabla}_{[X, Y]} Z\right)_{\eta}, \quad \eta \in \mathcal{D}_{\mu, D}^{s}$

Then for $s>(n / 2)+2, \tilde{R}$ is right invariant and continuous in the $H^{s}$ topology.

Since the weak curvature operator $\tilde{R}$ is bounded in $H^{s}$ for $s>$ $\frac{n}{2}+2$, the fundamental existence and uniqueness theorem for ordinary differential equations provides us with the following:

Corollary 4. For $s>(n / 2)+2$ and $y, \dot{y} \in T_{e} \mathcal{D}_{\mu, D}^{s}$, there exists a unique $H^{s}$ vector field $Y(t)$ along a geodesic curve $\eta$ of $\langle\cdot, \cdot\rangle$ which is solution to the Jacobi equation

$$
\tilde{\nabla}_{\dot{\eta}} \tilde{\nabla}_{\dot{\eta}} Y+\tilde{R}_{\eta}(\dot{\eta}, Y) \dot{\eta}=0, \quad Y(0)=y, \quad \tilde{\nabla}_{\dot{\eta}} Y(0)=\dot{y} .
$$

Because the geodesic flow $\eta$ of the right invariant metric $\langle\cdot, \cdot\rangle$ on $\mathcal{D}_{\mu, D}^{s}$ is the solution of (1.3), and since the Jacobi equation is the linearization of the geodesic flow, Corollary 4 proves existence and uniqueness of solutions to the linearized LAE- $\alpha$ equations, where the linearization is carried-out about a solution $u=\partial_{t} \eta \circ \eta^{-1}$ to (1.4). We are thus able to follow Arnold [3], and study the Lagrangian stability of our solutions, by studying the curvature of our infinite-dimensional group. Positive curvature indicates stable motion, while negative curvature implies exponential divergence of trajectories.

Since this system, averages over the small-scale fluid motion, one might hope that solutions of (1.4) have nicer stability properties than solutions to the Euler equations. Geometrically, this implies that as $\alpha$ is increased away from zero, the sectional curvatures which are negative for the Euler flow, may flip sign and become positive. Indeed, this seems to be the case; we give a simple example. 
We consider periodic two-dimensional motion, so the configuration space is the group of volume-preserving diffeomorphisms of the twotorus $\mathbb{T}^{2}$. Consider the parallel sinusoidal steady flow given by the stream function $\xi=\cos (k, x)$ and let $\psi$ be any other vector of the tangent space at $e$, i.e., $\psi=\sum x_{l} e_{l}$, where $x_{-l}=\bar{x}_{l}$. Theorem 3.4 of [4] states that the curvature of the group $\mathcal{D}_{\mu}\left(T^{2}\right)$ in any two-dimensional plane containing the direction $\xi$ is non-positive and is given by

$$
K_{\xi \psi}=\frac{S}{4} \sum_{l} a_{k l}^{2}\left|x_{l}+x_{l+2 k}\right|^{2},
$$

where $a_{k l}=\frac{(k \times l)^{2}}{|k+l|}, k \times l=k_{1} l_{2}-k_{2} l_{1}$ is the (oriented) area of the parallelogram spanned by $k$ and $l$, and $S$ is the area of the torus. Then, a corollary of this theorem states that the curvature in the plane defined by the stream functions $\xi=\cos (k, x)$ and $\psi=\cos (l, x)$ is

$$
K_{\xi \psi}=-\left(k^{2}+l^{2}\right) \sin ^{2} \beta \sin ^{2} \gamma / 4 S,
$$

where $\beta$ is the angle between $k$ and $l$, and $\gamma$ is the angle between $k+l$ and $k-l$. Recall that these are the curvatures with respect to the right invariant $L^{2}$ metric.

Now using the right invariant metric $\langle\cdot, \cdot\rangle$ on $\mathcal{D}_{\mu}^{s}\left(\mathbb{T}^{2}\right)$, we can prove the following result [32]. Let $\tilde{K}(\xi, \psi)$ denote the sectional curvature on $\mathcal{D}_{\mu}^{s}\left(\mathbb{T}^{2}\right)$ with the right invariant metric $\langle\cdot, \cdot\rangle$, where $\xi=\cos (k, x)$ and $\psi=\cos (l, x)$. For $|\epsilon|$ sufficiently small, let $l=k+\epsilon$. Then for any $k$, there exists $0<\alpha_{0}(k)<1$, such that for all $\alpha>\alpha_{o}(k), \tilde{K}(\xi, \psi)>0$. This means that for certain large enough values of $\alpha$, the sectional curvature does indeed become positive, and stabilization of the flow occurs.

\section{Review of the Hilbert manifold of maps and diffeomorphism groups}

Let us briefly recall some facts concerning the geometry of the manifold of maps between two Riemannian manifolds. We refer the reader to [31], [14], and [15] for a comprehensive treatment of this subject. Let $(M, g)$ be a $C^{\infty}$ compact oriented $n$-dimensional Riemannian manifold with boundary, and let $(N, h)$ denote a $p$-dimensional compact oriented boundaryless Riemannian manifold. By Sobolev's embedding theorem, when $s>n / 2+k$, the set of Sobolev mappings $H^{s}(M, N)$ is a subset of 
$C^{k}(M, N)$ with continuous inclusion, and so for $s>n / 2$, an $H^{s}$-map of $M$ into $N$ is pointwise well-defined. Mappings in the space $H^{s}(M, N)$ are those whose first $s$ distributional derivatives are square integrable in any system of charts covering the two manifolds.

For $s>n / 2$, the space $H^{s}(M, N)$ is a $C^{\infty}$ differentiable Hilbert manifold. Let exp: $T N \rightarrow N$ be the exponential mapping associated with $h$. Then for each $\phi \in H^{s}(M, N)$, the map $\omega_{\exp }: T_{\phi} H^{s}(M, N) \rightarrow H^{s}(M, N)$ is used to provide a differentiable structure which is independent of the chosen metric, where $\omega_{\exp }(v)=\exp \circ v$, and

$$
T_{\phi} H^{s}(M, N)=\left\{u \in H^{s}(M, T N) \mid \bar{\pi} \circ u=\phi\right\},
$$

where $\bar{\pi}: T N \rightarrow N$.

When $\partial M \neq \emptyset$, the set $H^{s}(M, M)$ is not a smooth manifold. We can, however, embed $\bar{M}$ into its double $\tilde{M}$, a compact boundaryless manifold of the same dimension, extending the metric $g$ to $\tilde{M}$. Using the above construction, we form the $C^{\infty}$ manifold $H^{s}(M, \tilde{M})$. Then for $s>(n / 2)+1$, the set

$$
\begin{gathered}
\mathcal{D}^{s}=\left\{\eta \in H^{s}(M, \tilde{M}) \mid \eta \text { is bijective }, \eta^{-1} \in H^{s}(M, \tilde{M}),\right. \\
\eta \text { leaves } \partial M \text { invariant }\}
\end{gathered}
$$

is an open subset of $H^{s}(M, \tilde{M})$. By choosing a metric on $\tilde{M}$ for which $\partial M$ is a totally geodesic submanifold, the above construction provides $\mathcal{D}^{s}$ with a $C^{\infty}$ differentiable structure (see [13] for details). For each $\eta \in \mathcal{D}^{s}$, the tangent space at $\eta$ is given by

$$
T_{\eta} \mathcal{D}^{s}=\left\{u \in H^{s}(M, T M) \mid \pi \circ u=\eta, \quad g\left(u \circ \eta^{-1}, n\right)=0 \text { on } \partial M\right\}
$$

and the vector space $T_{e} \mathcal{D}^{s}$ consists of the $H^{s}$ class vector fields on $M$ which are tangent to $\partial M$.

Let $\mu$ denote the Riemannian volume form on $M$, and let

$$
\mathcal{D}_{\mu}^{s}:=\left\{\eta \in \mathcal{D}^{s} \mid \eta^{*}(\mu)=\mu\right\}
$$

be the subset of $\mathcal{D}^{s}$ whose elements preserve $\mu$. As proven in [13], the set $\mathcal{D}_{\mu}^{s}$ is a $C^{\infty}$ subgroup of $\mathcal{D}^{s}$ for $s>(n / 2)+1$. We call $\mathcal{D}_{\mu}^{s}$ the group of volume preserving diffeomorphisms of class $H^{s}$. The tangent space at $\eta \in \mathcal{D}_{\mu}^{s}$ is given by

$$
\begin{gathered}
T_{\eta} \mathcal{D}_{\mu}^{s}=\left\{u \in H^{s}(M, T M) \mid \pi \circ u=\eta, \quad g\left(u \circ \eta^{-1}, n\right)=0 \text { on } \partial M,\right. \\
\left.\operatorname{div}\left(u \circ \eta^{-1}\right)=0\right\},
\end{gathered}
$$


so that the vector space $T_{e} \mathcal{D}_{\mu}^{s}$ consists of divergence-free $H^{s}$ class vector fields on $M$ that are tangent to $\partial M$.

We have the following standard composition lemma:

Lemma 1 ( $\omega$ and $\alpha$ lemmas). For $\eta \in \mathcal{D}^{s}$, right multiplication

$$
R_{\eta}: \mathcal{D}^{s} \rightarrow \mathcal{D}^{s}\left(H^{s} \rightarrow H^{s}\right), \zeta \mapsto \zeta \circ \eta, \text { is } C^{\infty},
$$

and for $\eta \in \mathcal{D}^{s+r}$, left multiplication

$$
L_{\eta}: \mathcal{D}^{s} \rightarrow \mathcal{D}^{s} \quad\left(H^{s} \rightarrow H^{s}\right), \zeta \mapsto \eta \circ \zeta, \text { is } C^{r} .
$$

Finally, the inverse map $\left(\eta \mapsto \eta^{-1}\right): \mathcal{D}^{s} \rightarrow \mathcal{D}^{s}$ is only $C^{0}$ and not even locally Lipschitz continuous. Thus, $\mathcal{D}^{s}$ and $\mathcal{D}_{\mu}^{s}$ are not Lie groups, but are $C^{\infty}$ topological groups with $C^{\infty}$ right translation.

\section{Proof of Theorem 1}

\subsection{The Neumann group $\mathcal{D}_{\mu, N}^{s}$}

We begin by first establishing the result for $\mathcal{D}_{\mu, N}^{s}$. We split the proof into three steps.

Step 1. Bundles over $\mathcal{D}_{\mu}^{s}$ and the transversal mapping theorem.

Recall that a smooth map between Hilbert manifolds $f: \mathcal{M}_{1} \rightarrow \mathcal{M}_{2}$ is transversal to a submanifold $\mathcal{M}_{3}$ of $\mathcal{M}_{2}$ if for all $m \in f^{-1}\left(\mathcal{M}_{3}\right)$,

$$
T f(m)\left(T_{m} \mathcal{M}_{1}\right)+T_{f(m)} \mathcal{M}_{3}=T_{f(m)} \mathcal{M}_{2}
$$

The transversal mapping theorem asserts that $f^{-1}\left(\mathcal{M}_{3}\right)$ is a submanifold of $\mathcal{M}_{1}$ if $f$ is transversal to $\mathcal{M}_{3}$.

Let us define the following infinite dimensional vector bundles over $\mathcal{D}_{\mu}^{s}$ :

$$
\begin{aligned}
\mathcal{F} & =H_{\eta}^{s-\frac{3}{2}}(T M \mid \partial M) \downarrow \mathcal{D}_{\mu}^{s}, \\
\mathcal{E} & =H_{\eta}^{s-\frac{3}{2}}(T \partial M) \downarrow \mathcal{D}_{\mu}^{s}, \\
\mathcal{G} & =\left[H_{\eta}^{s-\frac{3}{2}}(T M \mid \partial M)^{*} \otimes H_{\eta}^{s-\frac{3}{2}}(T \partial M)\right] \downarrow \mathcal{D}_{\mu}^{s} .
\end{aligned}
$$


For $x \in \partial M$, let $\Pi_{x}: T_{x} M \rightarrow T_{x} \partial M$ be the $g$-orthogonal projector, and define the section $\Pi: \mathcal{D}_{\mu}^{s} \rightarrow \mathcal{G}$ pointwise by $\Pi(\eta)(x)=\Pi_{\eta(x)}$, so that for all $\eta \in \mathcal{D}_{\mu}^{s}, \Pi(\eta): H_{\eta}^{s-3 / 2}(T M \mid \partial M) \rightarrow H_{\eta}^{s-3 / 2}(T \partial M)$. For $n \in H^{s-1 / 2}(N)$, define the section of $\mathcal{F}, h_{n}: \mathcal{D}_{\mu}^{s} \rightarrow \mathcal{F}$, by

$$
h_{n}(\eta)=\left.T \eta\right|_{\partial M} \cdot n \text {. }
$$

Finally, let $f_{n}: \mathcal{D}_{\mu}^{s} \rightarrow \mathcal{E}$ denote the section of $\mathcal{E}$ which is given by

$$
f_{n}=\Pi \circ h_{n} .
$$

Then, the set $\mathcal{D}_{\mu, N}^{s}$ is the inverse image of $f_{n}$ acting on the zero section of $\mathcal{E}$.

Lemma 2. The map $f_{n}: \mathcal{D}^{s} \rightarrow \mathcal{E}$ is $C^{\infty}$.

Proof. This follows from Lemma 4, the trace theorem, and the fact that $\Pi$ is smooth, as $g$ and $\partial M$ are $C^{\infty}$. q.e.d.

Hence, by the transversal mapping theorem, to show that $\mathcal{D}_{\mu, N}^{s}$ is a $C^{\infty}$ subgroup of $\mathcal{D}_{\mu}^{s}$, we shall prove that $f_{n}$ is a surjection; this will provide $\mathcal{D}_{\mu, N}^{s}$ with smooth differentiable structure. That $\mathcal{D}_{\mu, N}^{s}$ is a $C^{\infty}$ subgroup then follows from the fact that $\mathcal{D}_{\mu, N}^{s}$ is trivially closed under right composition.

\section{Step 2. The covariant derivative of $f_{n}$.}

We use the symbol $\bar{\nabla}$ to denote the weak Levi-Civita covariant derivative on sections of $\mathcal{F}$ and $\mathcal{G}$ (as obtained in Lemma 4). Following the methodology of Lemma 4, we compute that for all $\eta \in \mathcal{D}_{\mu}^{s}$ and $u \in T_{\eta} \mathcal{D}_{\mu}^{s}, \bar{\nabla}_{u} h_{n}(\eta) \in \mathcal{F}_{\eta}=H_{\eta}^{s-3 / 2}(T M \mid \partial M)$ is given by

$$
\bar{\nabla}_{u} h_{n}(\eta)=\nabla_{n} u
$$

where $\nabla$ denotes the Levi-Civita covariant derivative in $\eta^{*}(T M)$.

Next, we compute the covariant derivative of the section $\Pi$ of $\mathcal{G}$. We shall denote the metric tensor $g$ evaluated at the point $\eta(x)$ by $g_{\eta(x)}$. Using the fact that $g$ is covariantly constant, and letting $(\cdot)^{\tan }$ denote the tangential component of a mapping $v: \partial M \rightarrow T M \mid \partial M$, we have that

$$
\begin{aligned}
g_{\eta(x)}\left(\left[\nabla_{u} \Pi_{\eta(x)}\right] \cdot v(x), z(x)\right)= & -g_{\eta(x)}\left(\left(\nabla_{u} v(x)\right)^{\tan }, z(x)\right) \\
& -g_{\eta(x)}\left(\left(\nabla_{u} z(x)\right)^{\tan }, v(x)\right) \\
& -u\left[g_{\eta(x)}\left(v^{\tan }(x), z^{\tan }(x)\right)\right],
\end{aligned}
$$


where we use the notation: $u[f]=d f \cdot u$ for any function $f \in C^{1}(M)$. It is clear that the operator $\nabla_{u} \Pi_{\eta}$ is self-adjoint with respect to $g$. By definition of the $g$-orthogonal projector $\Pi_{\eta(x)}$, we see that for all $x \in \partial M$,

$$
g_{\eta(x)}\left(\Pi_{\eta(x)} \cdot w(x), \nu(x)\right)=0, \quad \forall w \in \mathcal{F}_{\eta}, \nu \in H_{\eta}^{s-3 / 2}(N),
$$

so that setting the map $v$ in equation (4.1) equal to the mapping $\nu$, and noting that the covariant derivative $\bar{\nabla}$ on $\mathcal{G}$ is the functorial lift of $\nabla$, we obtain the formula

$$
\left[\bar{\nabla}_{u} \Pi(\eta)\right](\nu)=-\left(\nabla_{u} \nu\right)^{\tan }=S_{\nu}(u) .
$$

It follows that for all $\eta \in f_{n}^{-1}(0)$,

$$
\begin{aligned}
\bar{\nabla}_{u} f_{n}(\eta) & =\bar{\nabla}_{u} \Pi_{\eta} \cdot h(\eta)+\Pi_{\eta} \bar{\nabla}_{u} h(\eta) \\
& =S_{\nu}(u)+\left(\nabla_{n} u\right)^{\tan } \in \mathcal{E}_{\eta},
\end{aligned}
$$

where $\nu=\left.T \eta\right|_{\partial M} \cdot n \in H_{\eta}^{s-\frac{3}{2}}(N)$.

\section{Step 3. $f_{n}$ is a surjection.}

It remains to show that for all $\eta \in f_{n}^{-1}(0), \bar{\nabla} f_{n}(\eta): T_{\eta} \mathcal{D}_{\mu}^{s} \rightarrow \mathcal{E}_{\eta}$ is onto. Because right translation on $\mathcal{D}_{\mu}^{s}$ is a smooth operation, it suffices to find $u \in T_{e} \mathcal{D}_{\mu}^{s}$ such that $\bar{\nabla}_{u} f_{n}(e)=w$ for any $w \in H^{s-3 / 2}(T \partial M)$. To do so, we shall solve the following elliptic boundary value problem: Find $(u, p) \in T_{e} \mathcal{D}_{\mu}^{s} \times H^{s-1}(M) / \mathbb{R}$ such that

$$
\begin{gathered}
\left(1-\triangle_{r}\right) u+\operatorname{grad} p=F, \quad \operatorname{div} u=0 \text { in } M, \\
g(u, n)=0, \quad\left(\nabla_{n} u\right)^{\tan }+S_{n}(u)=w \text { on } \partial M,
\end{gathered}
$$

where $F \in H^{s-2}(T M), w \in H^{s-3 / 2}(T \partial M), n \in H^{s-1 / 2}(N)$.

We first define the space

$$
H_{A}^{1}(T M)=\left\{v \in H^{1}(T M) \mid \operatorname{div} v=0 \text { and } g(u, n)=0 \text { on } \partial M\right\},
$$

and establish the existence of a unique weak solution $u \in H_{A}^{1}(T M)$ to (4.2). Let $B: H_{A}^{1}(T M) \times H_{A}^{1}(T M) \rightarrow \mathbb{R}$ be the bilinear form given by

$$
B(u, v)=\int_{M}[g(u, v)+2 \bar{g}(\operatorname{Def} u, \operatorname{Def} v)] \mu .
$$

$B$ is symmetric and by Korn's inequality, which states that $|u|_{1} \leq$ $C \mid$ Def $\left.u\right|_{0}+C|u|_{0}$ (see, for example, [38] Corollary 12.3), there exists 
$\beta>0$ such that $\beta|u|_{1} \leq B(u, u)$; hence, $B$ is coercive with respect to $H_{A}^{1}(T M)$. Let $\mathfrak{F}: H_{A}^{1}(T M) \rightarrow \mathbb{R}$ be given by

$$
\mathfrak{F}(v)=\int_{M} g(F, v) \mu+\int_{\partial M} g(w, v) \mu_{\partial} .
$$

By the trace theorem,

$$
\left|\int_{\partial M} g(w, v) \mu_{\partial}\right| \leq C|w|_{L^{2}(T \partial M)}|v|_{1}
$$

so that together with the Cauchy-Schwartz inequality and the embedding $H^{1} \hookrightarrow L^{2}$, we see that $\mathfrak{F} \in H_{A}^{1}(T M)^{*}$. Hence, by the Lax-Milgram theorem, their exists a unique $u \in H_{A}^{1}(T M)$ satisfying $B(u, v)=\mathfrak{F}(v)$ for all $v \in H_{A}^{1}(T M)$. This, in turn, uniquely determines $p \in L^{2}(M) / \mathbb{R}$, as the solution of $B(u, v)-\mathfrak{F}(v)=\int_{M} p \cdot \operatorname{div} v \mu$ for all $v \in H^{1}(T M)$ that satisfy $g(u, n)=0$ on $\partial M$. We have thus obtained a unique weak solution $(u, p) \in H_{A}^{1}(T M) \times L^{2}(M) / \mathbb{R}$ of the boundary value problem (4.2).

Now, since

$$
2 \operatorname{Def}^{*} \operatorname{Def} u=-2 \operatorname{DivDef} u=-\triangle u-2 \operatorname{Ric}(u),
$$

we see that if $u \in H^{2}(T M) \cap H_{A}^{1}(T M)$ satisfies

$$
B(u, v)=\mathfrak{F}(v), \quad \forall v \in H_{A}^{1}(T M),
$$

then $\mathrm{u}$ is a solution of (4.2). We shall use an elliptic regularity argument to prove that $u$ is in fact a classical $H^{s}$ solution of (4.2).

Let $(U, \phi)$ coordinate chart on $\bar{M}$, and $\chi \in C_{0}^{\infty}(U)$. Since

$$
\left(1-\triangle_{r}\right)(\chi u)=\chi\left(\left(1-\triangle_{r}\right) u\right)+\left[\left(1-\triangle_{r}\right), \chi\right] u,
$$

and since $\left[\left(1-\triangle_{r}\right), \chi\right] u$ is a first-order differential operator, our elliptic regularization of $u$ can be localized to the chart $U$. We can assume that $U$ intersects $\partial M$, for otherwise, standard interior regularity estimates can be applied. Let $x^{i}$ denote the coordinates on $U$ and set $\partial_{i}=\partial / \partial x^{i}$. We may express the Hodge Laplacian $\triangle$ on $U$ as

$$
\triangle u=\triangle_{\mathrm{loc}}+Y(u)
$$

where $\triangle_{\text {loc }}=g^{i j}(x) \partial_{i} \partial_{j} u$, and $Y$ is a first order differential operator. 
We consider the boundary value problem in $U$ given by

$$
\begin{gathered}
\left(1-\triangle_{\mathrm{loc}}\right) u+\operatorname{grad} p=F, \quad \operatorname{div} u=\rho \text { in } U \\
B_{0}(u)=0, B_{1}(u)=w \text { on } \partial U
\end{gathered}
$$

where $B_{0}(u)=g(u, n), B_{1}(u)=2\left[\left(D u+D u^{t}\right) \cdot n\right]^{\tan }$, and $D u \cdot n=$ $\partial_{j} u^{i} g_{k}^{j} n^{k}$. Applying induction to the usual difference quotient argument (see, for example, [38]) yields the elliptic estimate

$$
|u|_{s}+|p|_{s-1} \leq C\left(|F|_{s-2}+|\rho|_{s-1}+\left|B_{0}(u)\right|_{s-1 / 2}+\left|B_{1}(u)\right|_{s-3 / 2}\right) .
$$

Hence, the operator

$$
\begin{aligned}
\overline{\mathcal{L}}: H^{s} & \cap H_{A}^{1}(T U) \\
& \rightarrow H^{s-2}(T U) \oplus H^{s-1}(U) \oplus H^{s-1 / 2}(T \partial U) \oplus H^{s-3 / 2}(T \partial U)
\end{aligned}
$$

given by

$$
\overline{\mathcal{L}} u=\left(\left(1-\triangle_{\mathrm{loc}} u\right), \operatorname{div} u, B_{0}(u), B_{1}(u)\right)=(F, \rho, 0, w)
$$

has closed range, and since its adjoint has a trivial kernel, $\overline{\mathcal{L}}$ is an isomorphism (see also [26] for an alternative proof that $\overline{\mathcal{L}}$ is an isomorphism).

A simple computation verifies that along $\partial M$,

$$
2[\operatorname{Def} u \cdot n]^{\tan }=\left(\nabla_{n} u\right)^{\tan }+S_{n}(u) \forall u \in H_{A}^{1}(T M),
$$

so that on $\partial U$, [Def $u \cdot n]^{\text {tan }}$ differs from $B_{1}(u)$ by a linear combination of $C^{\infty}$ Christoffel maps, and we shall denote this difference by $\Gamma(u)$. Hence, the operator

$$
\begin{aligned}
\mathcal{L}: H^{s} & \cap H_{A}^{1}(T U) \\
& \rightarrow H^{s-2}(T U) \oplus H^{s-1}(U) \oplus H^{s-1 / 2}(T \partial U) \oplus H^{s-3 / 2}(T \partial U)
\end{aligned}
$$

given by

$$
\mathcal{L} u=\left(\left(1-\triangle_{r}\right) u, \operatorname{div} u, B_{0}(u),\left(\nabla_{n} u\right)^{\tan }+S_{n}(u)\right)
$$

differs from $\overline{\mathcal{L}} u$ by the operator $\mathcal{K} u=(Y(u)+\operatorname{Ric}(u), 0,0, \Gamma(u))$ which is compact by Rellich's theorem. Therefore, $\mathcal{L}$ has index 0 and trivial kernel, and is thus an isomorphism, which concludes that $\mathcal{D}_{\mu, N}^{s}$ is a $C^{\infty}$ subgroup of $\mathcal{D}_{\mu}^{s}$.

With an almost trivial modification, $\mathcal{D}_{N}^{s}$ is a $C^{\infty}$ subgroup of $\mathcal{D}^{s}$. To see this, we redefine the vector bundles $\mathcal{E}, \mathcal{F}, \mathcal{G}$ to have $\mathcal{D}^{s}$ as base 
manifold rather than $\mathcal{D}_{\mu}^{s}$, and we redefine the space $H_{A}^{1}(T M)$, removing the divergence-free constraint. In this case,

$$
2 \operatorname{Def}^{*} \operatorname{Def} u=-2 \operatorname{DivDef} u=-(\triangle+2 \text { Ric }+\operatorname{grad} \operatorname{div}) u,
$$

so to establish that $f_{n}$ is a surjection, we solve the following boundary value problem: For $F \in H^{s-2}(T M), w \in H^{s-3 / 2}(T \partial M)$ and $n \in$ $H^{s-1 / 2}(N)$, find $u \in T_{e} \mathcal{D}^{s}$ satisfying

$$
\begin{gathered}
{\left[1-\left(\triangle_{r}+\operatorname{grad} \operatorname{div}\right)\right] u=F \text { in } M} \\
g(u, n)=0, \quad\left(\nabla_{n} u\right)^{\tan }+S_{n}(u)=w \text { on } \partial M .
\end{gathered}
$$

A weak solution in $H_{A}^{1}(T M)$ is obtained using the Lax-Milgram theorem just as in Step 3 above. Up to a compact operator, this is precisely the elliptic system studied in ([16]), wherein existence and uniqueness of classical $H^{s}$ solutions is established. Since modification of an elliptic operator by lower-order terms does not change its index, we have existence of $u \in T_{e} \mathcal{D}_{\mu}^{s}$ solving (4.3), and this completes the argument for the subgroup $\mathcal{D}_{N}^{s}$.

\subsection{The mixed group $\mathcal{D}_{\mu, \text { mix }}^{s}$}

We shall follow the three step proof above, keeping the same notation.

Step 1. Bundles over $\mathcal{D}_{\mu}^{s}$ and the inverse function theorem.

We modify the vector bundles $\mathcal{F}, \mathcal{E}$, and $\mathcal{G}$ as follows:

$$
\begin{aligned}
\mathcal{F} & =H_{\eta}^{s-\frac{3}{2}}\left(T M \mid \Gamma_{2}\right) \downarrow \mathcal{D}_{\mu}^{s}, \\
\mathcal{E} & =H_{\eta}^{s-\frac{3}{2}}\left(T \Gamma_{2}\right) \downarrow \mathcal{D}_{\mu}^{s}, \\
\mathcal{G} & =\left[H_{\eta}^{s-\frac{3}{2}}\left(T M \mid \Gamma_{2}\right)^{*} \otimes H_{\eta}^{s-\frac{3}{2}}\left(T \Gamma_{2}\right)\right] \downarrow \mathcal{D}_{\mu}^{s} .
\end{aligned}
$$

For $n \in H^{s-1 / 2}\left(N \mid \Gamma_{2}\right)$, define $\bar{f}_{n}: \mathcal{D}_{\mu}^{s} \rightarrow \mathcal{D}^{s-1 / 2}\left(\Gamma_{1}\right) \times \mathcal{E}$ by

$$
\bar{f}_{n}(\eta)=\left[\left.\eta\right|_{\Gamma_{1}}, f_{n}(\eta)\right]=\left[\left.\eta\right|_{\Gamma_{1}}, \Pi(\eta) \circ\left(\left.T \eta\right|_{\Gamma_{2}} \cdot n\right)\right] .
$$

The trace theorem together with Lemma 2 ensures that $\bar{f}_{n}$ is $C^{\infty}$. Since $\mathcal{D}_{\mu, \text { mix }}^{S}=\bar{f}_{n}^{-1}(e, 0)$, we must prove that $\bar{f}_{n}$ is a surjection, in order to show that $\mathcal{D}_{\mu, \text { mix }}^{S}$ is a submanifold of $\mathcal{D}_{\mu}^{s}$. Again, it is clear that the set $\mathcal{D}_{\mu, \text { mix }}^{S}$ is closed under right composition.

Step 2. Computing the tangent map of $\bar{f}_{n}$. 
Step 2 of the case $\mathcal{D}_{\mu, N}^{s}$ shows that for any $u \in T_{\eta} \mathcal{D}_{\mu}^{s}$,

$$
\bar{\nabla}_{u} f_{n}=S_{\nu}(u)+\left(\nabla_{n} u\right)^{\tan } \in \mathcal{E}_{\eta}, \quad \nu=\left.T \eta\right|_{\Gamma_{2}} \cdot n \in H_{\eta}^{s-3 / 2}\left(N \mid \Gamma_{2}\right) .
$$

Now $\bar{\nabla}_{u} f_{n}$ is the vertical component of $T f_{n} \cdot u$, the $T \mathcal{E}$-valued image of $u$ under the tangent mapping $T f_{n}$. Letting $\mathcal{H} \subset T \mathcal{E}$ denote the connection associated with the Levi-Civita covariant derivative $\bar{\nabla}$ (see Step 1 above), we have the local decomposition $T f_{n} \cdot u=\bar{\nabla}_{u} f_{n}-\omega_{\mathcal{H}}(u)$. $f_{n}$, where $\omega_{\mathcal{H}}$ is the local connection 1 -form on $\mathcal{E}$ associated with the horizontal distribution $\mathcal{H}$. Then,

$$
T \bar{f}_{n}(\eta) \cdot u=\left(\left.u\right|_{\Gamma_{1}}, \bar{\nabla}_{u} f_{n}(\eta)-\omega_{\mathcal{H}}(u) \cdot f_{n}(\eta)\right), \quad u \in T_{\eta} \mathcal{D}_{\mu}^{s} .
$$

\section{Step 3. $\bar{f}_{n}$ is a surjection.}

It suffices to prove that for all $(\psi, w) \in H^{s-1 / 2}\left(T M \mid \Gamma_{1}\right) \times \mathcal{E}_{e}$, there exists $u \in T_{e} \mathcal{D}_{\mu}^{s}$ such that

$$
\begin{aligned}
u & =\psi \text { on } \Gamma_{1} \\
\left(\nabla_{n} u\right)^{\tan }+S_{n}(u) & =w \text { on } \Gamma_{2},
\end{aligned}
$$

and to do so, we shall follow Step 3 for the case of $\mathcal{D}_{\mu, N}^{s}$, and obtain $u$ as the solution of

$$
\begin{gathered}
\left(1-\triangle_{r}\right) u+\operatorname{grad} p=F, \quad \operatorname{div} u=0, \text { in } M, \\
u=\psi \text { on } \Gamma_{1}, \\
g(u, n)=0, \quad\left(\nabla_{n} u\right)^{\tan }+S_{n}(u)=w \text { on } \Gamma_{2} .
\end{gathered}
$$

It suffices to consider the homogeneous boundary condition $u=0$ on $\Gamma_{1}$.

To obtain a weak solution to (4.4), we define

$$
\begin{array}{r}
H_{A}^{1}(T M)=\left\{v \in H^{1}(T M) \mid \operatorname{div} v=0, g(u, n)=0 \text { on } \Gamma_{2}\right. \\
\text { and } \left.u=0 \text { on } \Gamma_{1}\right\},
\end{array}
$$

and again consider the bilinear form $B: H_{A}^{1}(T M) \times H_{A}^{1}(T M) \rightarrow \mathbb{R}$ given by

$$
B(u, v)=\int_{M}[g(u, v)+2 \bar{g}(\operatorname{Def} u, \operatorname{Def} v)] \mu .
$$

We define $\mathfrak{F}: H_{A}^{1}(T M) \rightarrow \mathbb{R}$ by

$$
\mathfrak{F}(v)=\int_{M} g(F, v) \mu+\int_{\Gamma_{2}} g(w, v) \mu_{\partial} .
$$


The argument we gave in Step 3 of the case $\mathcal{D}_{\mu, N}^{s}$ shows that there exists a unique solution $u \in H_{A}^{1}(T M)$ satisfying $B(u, v)=\mathfrak{F}(v)$ for all $v \in H_{A}^{1}(T M)$.

Now, if $u \in H^{2}(T M) \cap H_{A}^{1}(T M)$ satisfies $B(u, v)=\mathfrak{F}(v)$ for all $v \in H_{A}^{1}(T M)$, then $u$ is a solution of the mixed problem (4.4) for which elliptic regularity is slightly more subtle than for the Neumann problem. In particular, the identical argument which we used for that problem provides the $H^{s}$ class regularity of $u$ on $M /\left(\Gamma_{1} \cap \Gamma_{2}\right)$; after all, the boundary conditions on both $\Gamma_{1}$ and $\Gamma_{2}$ are elliptic in the sense of Agmon-Douglis-Nirenberg as the Complementary Condition is satisfied (see [1], and see [37] for an alternative method). The fact that $\partial M$ is $C^{\infty}$ and that $\partial M=\Gamma_{1} \cup \Gamma_{2}$ gives the regularity of the solution on $\bar{M}$ (see, for example, Fichera [16], pages 377 and 385). Hence, our argument in Step 3 for the subgroup $\mathcal{D}_{\mu, N}^{s}$ given above yields a unique solution $u \in H^{s}(T M) \cap H_{A}^{1}(T M)$ of (4.4), and thus concludes the proof that $\mathcal{D}_{\mu, \text { mix }}^{s}$ is a $C^{\infty}$ subgroup of $\mathcal{D}_{\mu}^{s}$.

Just as we proved that $\mathcal{D}_{N}^{s}$ is a subgroup of $\mathcal{D}^{s}$ by a minor modification of the argument for the case $\mathcal{D}_{\mu, N}^{s}$, we easily obtain that $\mathcal{D}_{\text {mix }}^{s}$ is also a $C^{\infty}$ subgroup of $\mathcal{D}^{s}$.

\subsection{The subgroup $\mathcal{D}_{\mu, D}^{s}$}

This case was studied by Ebin-Marsden [13] using a different approach. By setting $\Gamma_{2}=\emptyset$ above, we immediately prove that $\mathcal{D}_{\mu, D}^{s}$ is a $C^{\infty}$ subgroup of $\mathcal{D}_{\mu}^{s}$ and that $\mathcal{D}_{D}^{s}$ is a $C^{\infty}$ subgroup of $\mathcal{D}^{s}$, with the appropriate tangent spaces at the identity.

This concludes the proof of Theorem 1 .

\subsection{The group exponential map.}

Let $\mathfrak{G}^{s}$ denote either of the groups $\mathcal{D}_{D}^{s}, \mathcal{D}_{N}^{s}$, or $\mathcal{D}_{\text {mix }}^{s}$, and similarly, let $\mathfrak{G}_{\mu}^{s}$ denote either of the groups $\mathcal{D}_{\mu, D}^{s}, \mathcal{D}_{\mu, N}^{s}$, or $\mathcal{D}_{\mu, m i x}^{s}$.

Corollary 5. Let $V \in T_{e} \mathfrak{G}^{s}$, and let $\eta_{t}$ be its flow, $(d / d t) \eta_{t}=$ $V \circ \eta_{t}$. Then, for $s>(n / 2)+2, \eta_{t}$ is a one parameter subgroup of $\mathcal{G}^{s}$, and the group exponential map Exp: $T_{e} \mathfrak{G}^{s} \rightarrow \mathfrak{G}^{s}$ given by $V \mapsto \eta_{1}$ is continuous but not continuously differentiable, while the curve $t \mapsto \eta_{t}$ is $C^{1}$. This holds for $\mathfrak{G}_{\mu}^{s}$ as well.

Proof. The result follows from ([13], Theorems 3.1 and 6.3). $\quad$ q.e.d. 


\subsection{Further remarks on diffeomorphism subgroups}

The existence of the above $C^{\infty}$ subgroups follows from the existence, uniqueness, and regularity of solutions to certain elliptic boundary value problems.

This methodology allows to prove directly that for $s>(n / 2)+1$, $\mathcal{D}_{\mu, m i x}^{s}$ is a $C^{\infty}$ subgroup of $\mathcal{D}^{s}$.

We need only modify the map $\bar{f}_{n}$ given in Step 3 above as follows: For $n \in H^{s-1 / 2}\left(N \mid \Gamma_{2}\right)$ and $\mu$ the Riemannian volume form on $M$, define $\bar{f}_{n, \mu}: \mathcal{D}_{\mu}^{s} \rightarrow \Lambda^{3}(M) \times \mathcal{D}^{s-1 / 2}\left(\Gamma_{1}\right) \times \mathcal{E}$ by

$$
\bar{f}_{n, \mu}(\eta)=\left[\eta^{*}(\mu),\left.\eta\right|_{\Gamma_{1}}, \Pi(\eta) \circ\left(\left.T \eta\right|_{\Gamma_{2}} \cdot n\right)\right] .
$$

Again $\bar{f}_{n, \mu}$ is $C^{\infty}$, and following the notation of Step 2, we easily compute that

$T \bar{f}_{n, \mu}(\eta) \cdot u=\left(\operatorname{div}\left(u \circ \eta^{-1}\right),\left.u\right|_{\Gamma_{1}}, \bar{\nabla}_{u} f_{n}(\eta)-\omega_{\mathcal{H}}(u) \cdot f_{n}(\eta)\right), u \in T_{\eta} \mathcal{D}_{\mu}^{s}$.

Finally, the modification to Step 3 consists of obtaining a solution $u \in T_{e} \mathcal{D}_{\mu}^{s}$ satisfying the boundary value problem

$$
\begin{gathered}
\left(1-\triangle_{r}\right) u+\operatorname{grad} p=F, \quad \operatorname{div} u=q, \text { in } M, \\
u=\psi \text { on } \Gamma_{1}, \\
g(u, n)=0, \quad\left(\nabla_{n} u\right)^{\tan }+S_{n}(u)=w \text { on } \Gamma_{2} .
\end{gathered}
$$

Only minor modifications need be made to our previous proofs, so we leave this for the interested reader.

Of course, setting $\Gamma_{2}=\emptyset$ proves the theorem when $\mathcal{D}_{\mu, \text { mix }}^{s}$ is replaced by $\mathcal{D}_{\mu, D}^{s}$, while setting $\Gamma_{1}=\emptyset$ proves the theorem in the case that $\mathcal{D}_{\mu, \text { mix }}^{s}$ is replaced by $\mathcal{D}_{\mu, N}^{s}$.

\section{The Stokes decomposition for manifolds with boundary}

In this section we recall well-known results about the Hodge decomposition for manifolds with boundary (see [12] and [28] for proofs), and define a new Stokes decomposition based on the solution to the Stokes problem, whose summands are $\langle\cdot, \cdot\rangle_{e}$-orthogonal.

Let $(M, g)$ be a $C^{\infty}$ compact, oriented Riemannian $n$-dimensional manifold with $C^{\infty}$ boundary $\partial M$, and let $i: \partial M \rightarrow M$ be the inclusion map. Then for a smooth vector field $X$ on $M$ and $n$, the outwardpointing normal vector field on $\left.\partial M, i^{*}(X\lrcorner \mu\right)=g(X, n) \mu_{\partial}$ where $\mu$ is 
the Riemannian volume form, and $\mu_{\partial}$ is the volume form on $\partial M$ coming from the induced Riemannian metric.

By the trace theorem, $i^{*} \alpha$ is well-defined on $\partial M$ for $\alpha \in H^{s}\left(\Lambda^{k}(M)\right)$ when $s \geq 1$; hence, for such $s, \alpha \in H^{s}\left(\Lambda^{k}(M)\right)$ is tangent (\|) to $\partial M$ if and only if $n\lrcorner \alpha=0$, and normal $(\perp)$ to $\partial M$ if and only if $n^{\sharp} \wedge \alpha=0$.

When $\partial M=\emptyset,(d \alpha, \beta)_{L^{2}}=(\alpha, d \beta)_{L^{2}}$, where $(\phi, \psi)_{L^{2}}=\int_{M} \phi \wedge * \psi$ (here, $*: \Lambda^{k}(M) \rightarrow \Lambda^{n-k}(M)$ denotes the Hodge star operator), and we have the standard Hodge decomposition

$$
H^{s}\left(\Lambda^{k}\right)=d\left(H^{s+1}\left(\Lambda^{k-1}\right)\right) \oplus \delta\left(H^{s+1}\left(\Lambda^{k+1}\right)\right) \oplus \mathcal{H}^{s, k},
$$

where $\mathcal{H}^{s, k}=\left\{\alpha \in H^{s}\left(\Lambda^{k}(M)\right) \mid d \alpha=0\right.$ and $\left.\delta \alpha=0\right\}$ are the Harmonic fields.

When $\partial M \neq \emptyset$, we have that

$$
(d \alpha, \beta)_{L^{2}}-(\alpha, \delta \beta)_{L^{2}}=\int_{\partial M}\left(n^{\sharp} \wedge \alpha, \beta\right) \mu_{\partial}
$$

and

$$
\left.(\delta \alpha, \beta)_{L^{2}}-(\alpha, d \beta)_{L^{2}}=-\int_{\partial M}(n\lrcorner \alpha, \beta\right) \mu_{\partial} .
$$

This shows that if $\delta \alpha=0$, then $\alpha \| \partial M$ iff $(\alpha, d \beta)_{L^{2}}=0$ for all $\beta$, in which case the notion of $\alpha \| \partial M$ is well-defined even if $\alpha$ is only of class $L^{2}$. Similarly, if $d \alpha=0$, then $\alpha \perp \partial M$ iff $(\alpha, d \beta)=0$ for all $\beta$. We define

$$
\begin{aligned}
H_{t}^{s}\left(\Lambda^{k}\right) & =\left\{\alpha \in H^{s}\left(\Lambda^{k}(M)\right) \mid \alpha \| \partial M\right\}, \\
H_{n}^{s}\left(\Lambda^{k}\right) & =\left\{\alpha \in H^{s}\left(\Lambda^{k}(M)\right) \mid \alpha \perp \partial M\right\}, \\
\mathcal{H}_{t}^{s, k} & =\left\{\alpha \in \mathcal{H}^{s} \mid \alpha \| \partial M\right\}, \\
\mathcal{C}_{t}^{s, k} & =\left\{\alpha \in H^{s}\left(\Lambda^{k}(M)\right) \mid \delta \alpha=0 \text { and } \alpha \| \partial M\right\} .
\end{aligned}
$$

Then for $s \geq 0$, we have the Hodge decompositions

$$
\begin{aligned}
& H^{s}\left(\Lambda^{k}\right)=d\left(H_{n}^{s+1}\left(\Lambda^{k-1}\right)\right) \oplus \delta\left(H_{t}^{s+1}\left(\Lambda^{k+1}\right)\right) \oplus \mathcal{H}^{s, k}, \\
& H^{s}\left(\Lambda^{k}\right)=d\left(H^{s+1}\left(\Lambda^{k-1}\right)\right) \oplus \mathcal{C}_{t}^{s, k},
\end{aligned}
$$

from which we can define the $L^{2}$ orthogonal projection onto $\operatorname{ker}(\delta)$.

Consider the Hodge Laplacian $-\triangle=\delta d+d \delta$ with domain

$$
\left.\left.\left\{\alpha \in H^{2}\left(\Lambda^{k}(M)\right) \mid n\right\lrcorner \alpha=0 \text { and } n\right\lrcorner d \alpha=0\right\},
$$


and let $\mathfrak{P}_{t}$ denote the $L^{2}$ orthogonal projection onto $\mathcal{H}_{t}^{s, k}$. We call

$$
\begin{gathered}
P_{e}: H^{s}\left(\Lambda^{k}\right) \rightarrow H_{t}^{s}\left(\Lambda^{k}\right) \\
P_{e}(\omega)=\mathfrak{P}_{t} \omega+\delta d(-\triangle)^{-1}\left(\omega-\mathfrak{P}_{t} \omega\right)
\end{gathered}
$$

the $L^{2}$ Hodge projection.

We shall now restrict our attention to $H^{s}\left(\Lambda^{1}(M)\right)$ and identifying 1 -forms with vector fields thru the metric $g$ on $M$. Letting

$$
\mathcal{X}_{t}^{s}=\left\{u \in H^{s}(T M) \mid \operatorname{div} u=0, u \| \partial M\right\},
$$

we may equivalently express the Hodge decomposition as

$$
H^{s}(T M)=\operatorname{grad} H^{s+1}(M) \oplus \mathcal{X}_{t}^{s},
$$

so that for all $u \in H^{s}(T M), u=v+\operatorname{grad} p$, where $v \in \mathcal{X}_{t}^{s}$ and $p: M \rightarrow$ $\mathbb{R}$ is obtained as the solution of Neumann problem

$$
\begin{aligned}
\triangle p & =\operatorname{div} u \quad \text { in } M \\
g(\operatorname{grad} p, n) & =g(u, n) \quad \text { on } \partial M .
\end{aligned}
$$

Thus, a convenient and equivalent formula for the $L^{2}$ Hodge projection is

$$
P_{e}: H^{s}(T M) \rightarrow \mathcal{X}_{t}^{s}, \quad P_{e}(u)=u-\operatorname{grad} p .
$$

For each $\eta \in \mathcal{D}_{\mu}^{s}$, we define the projector

$$
\begin{gathered}
P_{\eta}: T_{\eta} \mathcal{D}^{s} \rightarrow T_{\eta} \mathcal{D}_{\mu}^{s}, \\
P_{\eta}(X)=\left(P_{e}\left(X \circ \eta^{-1}\right)\right) \circ \eta .
\end{gathered}
$$

Thus $\bar{P}: T \mathcal{D}^{s} \rightarrow T \mathcal{D}_{\mu}^{s}$, given on each fiber by $P_{\eta}$, is a bundle map covering the identity and is $C^{\infty}$ by Appendix A of [13].

Next, we define a new projector based on the elliptic Stokes problem. Let $\mathfrak{G}^{s}$ denote $\mathcal{D}_{D}^{s}, \mathcal{D}_{N}^{s}$, or $\mathcal{D}_{\text {mix }}^{s}$, and similarly, let $\mathfrak{G}_{\mu}^{s}$ denote $\mathcal{D}_{\mu, D}^{s}$, $\mathcal{D}_{\mu, N}^{s}$, or $\mathcal{D}_{\mu, m i x}^{s}$.

For $r \geq 1$, let $\mathcal{V}^{r}$ denote the $H^{r}$ vector fields on $M$ which satisfy the boundary conditions prescribed to elements of $T_{e} \mathfrak{G}^{s}$, and set $\mathcal{V}_{\mu}^{r}=$ $\left\{u \in \mathcal{V}^{r} \mid \operatorname{div} u=0\right\}$. If $1 \leq r<2$, then elements of $\mathcal{V}^{r}$ and $\mathcal{V}_{\mu}^{r}$ only satisfy the essential boundary conditions $\left(u=0\right.$ on $\partial M$ if $\mathfrak{G}_{\mu}^{s}=\mathcal{D}_{\mu, D}^{s}$, $g(u, n)=0$ on $\partial M$ if $\mathfrak{G}_{\mu}^{s}=\mathcal{D}_{\mu, N}^{s}$, or $u=0$ on $\Gamma_{1}$ and $g(u, n)=0$ on $\Gamma_{2}$ if $\mathfrak{G}_{\mu}^{s}=\mathcal{D}_{\mu, \text { mix }}^{s}$ ) because vector fields in $\mathcal{V}^{r}$ for $r<2$ do not possess sufficient regularity for the trace map to detect derivatives on the boundary.

We set $\mathcal{L}=-2$ Def* $^{*}$ Def, and consider the positive self-adjoint unbounded operator $(1-\mathcal{L})$ on $L^{2}(T M)$ with domain $D(1-\mathcal{L})=\mathcal{V}^{2}$. 
Proposition 1. For $r \geq 1$ we have the following well defined decomposition

$$
\mathcal{V}^{r}=\mathcal{V}_{\mu}^{r} \oplus(1-\mathcal{L})^{-1} \operatorname{grad} H^{r-1}(M) .
$$

Thus, if $F \in \mathcal{V}^{r}$, then there exists $(v, p) \in \mathcal{V}_{\mu}^{r} \times H^{r-1}(M) / \mathbb{R}$ such that

$$
F=v+(1-\mathcal{L})^{-1} \operatorname{grad} p
$$

and the pair $(v, p)$ are solutions of the Stokes problem

$$
\begin{gathered}
(1-\mathcal{L}) v+\operatorname{grad} p=(1-\mathcal{L}) F, \\
\operatorname{div} v=0, \\
v \text { satisfies boundary conditions } \\
\text { prescribed to elements of } \mathcal{V}^{r} .
\end{gathered}
$$

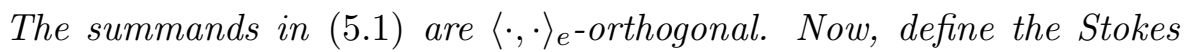
projector

$$
\begin{gathered}
\mathcal{P}_{e}: \mathcal{V}^{r} \rightarrow \mathcal{V}_{\mu}^{r}, \\
\mathcal{P}_{e}(F)=F-(1-\mathcal{L})^{-1} \operatorname{grad} p .
\end{gathered}
$$

Then, for $s>(n / 2)+1, \overline{\mathcal{P}}: T \mathfrak{G}^{s} \rightarrow T \mathfrak{G}_{\mu}^{s}$, given on each fiber by

$$
\begin{gathered}
\overline{\mathcal{P}}_{\eta}: T_{\eta} \mathfrak{G}^{s} \rightarrow T_{\eta} \mathfrak{G}_{\mu}^{s}, \\
\overline{\mathcal{P}}_{\eta}\left(X_{\eta}\right)=\left[\mathcal{P}_{e}\left(X_{\eta} \circ \eta^{-1}\right)\right] \circ \eta,
\end{gathered}
$$

is a $C^{\infty}$ bundle map covering the identity.

Proof. Acting on divergence-free vector-fields, $\mathcal{L}=\triangle_{r}$. Thus, the proof that $\mathcal{D}_{\mu, \text { mix }}^{s}$ is a $C^{\infty}$ subgroup of $\mathcal{D}_{\mu}^{s}$ shows that the Stokes problem (5.2) has a unique solution $(v, p) \in \mathcal{V}_{\mu}^{r} \times H^{r-1}(M) / \mathbb{R}$ for any $F \in \mathcal{V}^{r}$, $r \geq 1$.

It is easy to verify that the summands in $(5.1)$ are $\langle\cdot, \cdot\rangle_{e^{-}}$orthogonal, so it only remains to show that $\overline{\mathcal{P}}$ is smooth. For $F_{\eta} \in T_{\eta} \mathfrak{G}^{s}$, let $F=F_{\eta} \circ \eta^{-1}$, and let $(v, p)$ solve (5.2). By (5.3), it suffices to prove that

$$
\left[(1-\mathcal{L})^{-1} \operatorname{grad} p\right] \circ \eta=\left[(1-\mathcal{L})^{-1} \operatorname{grad} \triangle^{-1} \operatorname{div}(1-\mathcal{L})(v-F)\right] \circ \eta
$$

is smooth. Letting $V_{\eta}=v \circ \eta \in T_{\eta} \mathfrak{G}_{\mu}^{s}$, we have the equivalent expression for $\left[(1-\mathcal{L})^{-1} \operatorname{grad} p\right] \circ \eta$ given by

$$
{\overline{(1-\mathcal{L})^{-1}}}_{\eta} \circ \overline{\operatorname{grad}}_{\eta} \circ{\overline{\triangle^{-1}}}_{\eta} \circ \overline{\operatorname{div}}_{\eta} \circ{\overline{(1-\mathcal{L})_{\eta}}}_{\eta}\left(V_{\eta}-F_{\eta}\right)
$$

which is a $C^{\infty}$ bundle map by Proposition 5 together with Lemmas 5 and $6 . \quad$ q.e.d. 


\section{A new right invariant metric on $\mathcal{D}_{\mu, D}^{s}, \mathcal{D}_{\mu, N}^{s}, \mathcal{D}_{\mu, m i x}^{s}$ and its geodesics}

Recall that a weak Riemannian metric on a Hilbert manifold $\mathcal{M}$ is given by a map $\gamma$ which assigns to each $m \in \mathcal{M}$, a continuous positivedefinite symmetric bilinear form $\gamma(m) \in T_{m}^{*} \mathcal{M} \otimes T_{m}^{*} \mathcal{M}$, which is $C^{\infty}$ with respect to $m \in \mathcal{M}$. The metric $\gamma$ is termed weak, because it defines a topology which is weaker than the original topology on $\mathcal{M}$ (and hence on $\left.T_{m} \mathcal{M}\right)$.

In general, the geodesic flow of a weak metric does not exist. A simple example is given by the lack of a well-defined exponential map for the usual $L^{2}$ metric on $\mathcal{D}^{s}$ when $\partial M$ is not empty. Nevertheless, the seminal paper of Ebin-Marsden [13] proves that it is indeed possible to define a weak right invariant $L^{2}$ metric on $\mathcal{D}_{\mu}^{s}$ for manifolds with boundary, and that this weak metric induces a (weak) Levi-Civita covariant derivative and geodesic flow. As we have described, the geodesic flow of the invariant $L^{2}$ metric on $\mathcal{D}_{\mu}^{s}$ generates solutions to the Euler equations of ideal hydrodynamics; we shall introduce a new weak invariant metric on $\mathcal{D}_{\mu}^{s}$ which, remarkably, also generates the geodesic flow that solves the LAE- $\alpha$ equations.

Let $\mathfrak{G}_{\mu}^{s}$ denote either $\mathcal{D}_{\mu, D}^{s}, \mathcal{D}_{\mu, N}^{s}$, or $\mathcal{D}_{\mu, \text { mix }}^{s}$, and let $\bar{g}$ denote the induced inner-product on the fibers of $\left[T^{*} M \otimes T^{*} M\right]^{* \otimes 2}$.

Proposition 2. Define the bilinear form $\langle\cdot, \cdot\rangle_{e}$ on $T_{e} \mathfrak{G}_{\mu}^{s}$ as follows: for $X, Y \in T_{e} \mathfrak{G}_{\mu}^{s}$ and $\alpha>0$, set

$$
\begin{aligned}
\langle X, Y\rangle_{e} & =\int_{M}\left(g_{x}(X(x), Y(x))+\frac{\alpha^{2}}{2} \bar{g}_{x}\left(£_{X} g(x), £_{Y} g(x)\right)\right) \mu(x) \\
& =\int_{M}\left(g_{x}(X(x), Y(x))+2 \alpha^{2} \bar{g}_{x}(\operatorname{Def}(X)(x), \operatorname{Def}(Y)(x)) \mu(x)\right.
\end{aligned}
$$

and define a bilinear form on each fiber of $T \mathfrak{G}_{\mu}^{s}$ by right translation so that for $X_{\eta}, Y_{\eta} \in T_{\eta} \mathfrak{G}_{\mu}^{s}$,

$$
\left\langle X_{\eta}, Y_{\eta}\right\rangle_{\eta}=\left\langle X_{\eta} \circ \eta^{-1}, Y_{\eta} \circ \eta^{-1}\right\rangle_{e} .
$$

Then $\langle\cdot, \cdot\rangle$, given on each fiber by $\langle\cdot, \cdot\rangle_{\eta}$, is a right invariant weak Riemannian metric on $\mathfrak{G}_{\mu}^{s}$.

Proof. That $\langle\cdot, \cdot\rangle$ is $C^{\infty}$ on $\mathfrak{G}_{\mu}^{s}$ follows from Lemma 1. That $\langle\cdot, \cdot\rangle_{\eta}$ is a positive-definite symmetric bilinear form is proven as follows:

$$
2 \text { Def } u=£_{u} g=\nabla u+\nabla u^{t},
$$


so for any of the boundary conditions prescribed on elements of $T_{e} \mathfrak{G}_{\mu}^{s}$, we have that

$$
0 \leq 2 \operatorname{Def}^{*} \text { Def } u=-(\triangle+2 \operatorname{Ric}) u,
$$

so that integrating by parts (and noting that the boundary terms vanish), we may express $\langle\cdot, \cdot\rangle_{e}$ in the equivalent form

$$
\langle X, Y\rangle_{e}=\int_{M} g_{x}\left(\left(1-\triangle_{r}\right) X(x), Y(x)\right) \mu(x) .
$$

Since $\left(1-\triangle_{r}\right)$ is a self-adjoint positive operator (on $L^{2}$ vector fields that are divergence-free), this shows that $\langle\cdot, \cdot\rangle$ is a well defined $C^{\infty}$ weak invariant Riemannian metric on $\mathfrak{G}_{\mu}^{s}$. $\quad$ q.e.d.

The metric $\langle\cdot, \cdot\rangle$ is invariant under the action of $\mathfrak{G}_{\mu}^{s}$, so the subgroups of the volume preserving diffeomorphism group that we have constructed play the role of both configuration space as well as symmetry group (this is the massive particle relabeling symmetry of hydrodynamics). In order to formally establish the equations of geodesic motion of the invariant metric $\langle\cdot, \cdot\rangle$ on $\mathfrak{G}_{\mu}^{s}$ we shall make use of the Euler-Poincaré reduction theorem. The reader unfamiliar with this symmetry reduction procedure is referred to Appendix A for a brief discussion.

Proposition 3. Let the pair $\left(\mathfrak{G}_{\mu}^{s},\langle\cdot, \cdot\rangle\right)$ denote either $\mathcal{D}_{\mu, D}^{s}, \mathcal{D}_{\mu, N}^{s}$, or $\mathcal{D}_{\mu, m i x}^{s}$ together with the right invariant Riemannian metric defined in (6.1). Then, a curve $\dot{\eta}(t) \in T \mathfrak{G}_{\mu}^{s}$ is a geodesic of $\langle\cdot, \cdot\rangle$ if and only if its projection onto the fiber over the identity given by $u(t)=\dot{\eta}(t) \circ \eta(t)^{-1} \in$ $T_{e} \mathfrak{G}_{\mu}^{s}$ is a solution of

$$
\begin{gathered}
\partial_{t}\left(1-\alpha^{2} \triangle_{r}\right) u+\nabla_{u}\left(1-\alpha^{2} \triangle_{r}\right) u-\alpha^{2} \nabla u^{t} \cdot \triangle_{r} u=-\operatorname{grad} p, \\
\operatorname{div} u=0, \quad u(0)=u_{0},
\end{gathered}
$$

together with the boundary conditions

$$
\begin{aligned}
& u=0 \text { on } \partial M \quad \text { if } \quad \mathfrak{G}_{\mu}^{s}=\mathcal{D}_{\mu, D}^{s}, \\
& \left.\begin{array}{c}
g(u, n)=0 \\
\left(\nabla_{n} u\right)^{\tan }+S_{n}(u)=0
\end{array}\right\} \text { on } \partial M \quad \text { if } \quad \mathfrak{G}_{\mu}^{s}=\mathcal{D}_{\mu, N}^{s} \\
& \left.\left.\begin{array}{c}
u=0 \\
g(u, n)=0, \\
\left(\nabla_{n} u\right)^{\tan }+S_{n}(u)=0
\end{array}\right\} \quad \begin{array}{l}
\text { on } \Gamma_{1} \\
\text { on } \Gamma_{2}
\end{array}\right\} \quad \text { if } \quad \mathfrak{G}_{\mu}^{s}=\mathcal{D}_{\mu, \text { mix }}^{s}
\end{aligned}
$$

where grad $p$ is completely determined by the Stokes projector $\mathcal{P}_{e}$. 
Proof. From part (d) of Proposition 6, the reduced Lagrangian is given by $\langle\cdot, \cdot\rangle_{e}$, so that $\dot{\eta}(t)$ is a geodesic of $\langle\cdot, \cdot\rangle$ on $\mathfrak{G}_{\mu}^{s}$ if $u(t)=\dot{\eta}(t) \circ$ $\eta(t)^{-1}$ is a fixed point of the reduced action function (on an arbitrary interval $(a, b)) s: T_{e} \mathfrak{G}_{\mu}^{s} \rightarrow \mathbb{R}$ given by

$$
s(u)=\frac{1}{2} \int_{a}^{b}\langle u(t), u(t)\rangle_{e} d t
$$

Let $\epsilon \mapsto \eta^{\epsilon}$ be a smooth curve in $\mathfrak{G}_{\mu}^{s}$ such that $\eta^{0}=\eta$ and

$$
\left.(d / d \epsilon) \eta^{\epsilon}\right|_{\epsilon=0}=\delta \eta \in T_{\eta} \mathfrak{G}_{\mu}^{s}
$$

the map $t \mapsto \delta \eta(t)$ is the variation of the curve $\eta(t)$ on the interval $(a, b)$ and $\delta \eta(a)=\delta \eta(b)=0$. The curve $\epsilon \mapsto \eta^{\epsilon}$ induces a curve $\epsilon \mapsto u^{\epsilon}$ in the single fiber $T_{e} \mathfrak{G}_{\mu}^{s}$ such that $u^{0}=u$ and $\left.(d / d \epsilon) u^{\epsilon}\right|_{\epsilon=0}=\delta u$. The Euler-Poincaré reduction theorem gives the relation

$$
\delta u=\partial_{t}\left(\delta \eta \circ \eta^{-1}\right)+\left[\delta \eta \circ \eta^{-1}, u\right]_{e} .
$$

Computing the first variation of the action $s$, we have that

$$
\begin{aligned}
d s(u) & \cdot \delta u \\
= & \int_{a}^{b} \int_{M}\left(g(u, \delta u)+2 \alpha^{2} \bar{g}(\operatorname{Def} u, \operatorname{Def} \delta u)\right) \mu d t \\
= & \int_{a}^{b}\left[\int_{M} g\left(\left(1-\alpha^{2} \triangle_{r}\right) u, \delta u\right) \mu\right. \\
& \left.\quad+\alpha^{2} \int_{\partial M} g\left(\left(\nabla_{n} u\right)^{\tan }+S_{n}(u), \delta u\right) \mu_{\partial}\right] d t .
\end{aligned}
$$

Since $u$ and $\delta u$ satisfy the boundary conditions prescribed to elements of $T_{e} \mathfrak{G}_{\mu}^{s}$, the boundary term in the above equation vanishes, leaving only

$$
d s(u) \cdot \delta u=\int_{a}^{b} \int_{M} g\left(\left(1-\alpha^{2} \triangle_{r}\right) u, \partial_{t}\left(\delta \eta \circ \eta^{-1}\right)+\left[\delta \eta \circ \eta^{-1}, u\right]_{e}\right) \mu d t .
$$

Using the formula $[x, y]_{e}=\nabla_{y} x-\nabla_{x} y$ and integrating by parts, we 
obtain

$$
\begin{array}{r}
d s(u) \cdot \delta u \\
=\int_{a}^{b} \int_{M} g\left(\left(1-\alpha^{2} \triangle_{r}\right) \partial_{t} u+\nabla_{u}\left(1-\alpha^{2} \triangle_{r}\right) u\right. \\
\left.-\alpha^{2} \nabla u^{t} \cdot \triangle_{r} u, \delta \eta \circ \eta^{-1}\right) \mu d t \\
=\int_{a}^{b}\left\langle\partial_{t} u+\left(1-\alpha^{2} \mathcal{L}\right)^{-1}\left[\nabla_{u}\left(1-\alpha^{2} \triangle_{r}\right) u\right.\right. \\
\left.\left.-\alpha^{2} \nabla u^{t} \cdot \triangle_{r} u\right], \delta \eta \circ \eta^{-1}\right\rangle_{e} d t
\end{array}
$$

where again $\mathcal{L}=-2 \mathrm{Def}^{*}$ Def. Since right translation is an isomorphism, $\delta \eta \circ \eta^{-1} \in T_{e} \mathfrak{G}_{\mu}^{s}$ is arbitrary, so $u$ is a fixed point of $s$ iff

$$
\partial_{t} u+\mathcal{P}_{e}\left(\left(1-\alpha^{2} \mathcal{L}\right)^{-1}\left[\nabla_{u}\left(1-\alpha^{2} \triangle\right) u-\alpha^{2} \nabla u^{t} \cdot \triangle u\right]\right)=0,
$$

and this is precisely $(6.2)$, as $\left(1-\alpha^{2} \mathcal{L}\right) \partial_{t} u=\left(1-\alpha^{2} \triangle_{r}\right) \partial_{t} u$ since $\operatorname{div} \partial_{t} u=0 . \quad$ q.e.d.

In the next section, we prove Theorem 2 by establishing existence and uniqueness of geodesics of the invariant metric. The following simple lemma will play a fundamental role.

Lemma 3. For $s>(n / 2)+1$, let $u, v \in T_{e} \mathcal{D}_{\mu, D}^{s}$, and consider the unbounded self-adjoint operator $(1-\mathcal{L})$ on $L^{2}$ with domain $D(1-\mathcal{L})=$ $H^{2}(T M) \cap H_{0}^{1}(T M)$. Then

$$
\begin{aligned}
(1-\mathcal{L}) \nabla_{u} v= & \nabla_{u}\left(1-\triangle_{r}\right) v-\operatorname{div}\left[\nabla v \cdot \nabla u^{t}+\nabla v \cdot \nabla u\right] \\
& -\operatorname{grad} \operatorname{Tr}[\nabla u \cdot \nabla v]+\left(\nabla_{u} \operatorname{Ric}\right) \cdot v \\
& -\operatorname{grad} \operatorname{Ric}(u, v)-\operatorname{Tr}[\nabla(R(u, \cdot) v)+R(u, \cdot) \nabla v] .
\end{aligned}
$$

Proof. First notice that for $s>(n / 2)+1, \nabla_{u} v$ is an $H^{s-1}$ vector field on $M$ whose trace vanishes on $\partial M$; thus, it makes sense for the operator $(1-\mathcal{L})$ to act on $\nabla_{u} v$.

Recall that $\mathcal{L}=-(\triangle+2 \mathrm{Ric}+\operatorname{grad} \operatorname{div})$, so we begin by computing the commutator of $\left[-\triangle, \nabla_{u}\right]$. Let $\left\{e_{i}\right\}$ be a local orthonormal frame, and write the Hodge Laplacian $\triangle=-(d \delta+\delta d)$ acting on 1-forms (identified with vector fields) as $\triangle=\nabla_{e_{i}} \nabla_{e_{i}}+$ Ric, so that

$$
\triangle \nabla_{u} v=\nabla_{e_{i}} \nabla_{e_{i}}\left(\nabla_{u} v\right)-\operatorname{Ric}\left(\nabla_{u} v\right) .
$$


Using the definition of the Riemannian curvature operator, we compute that

$$
\begin{aligned}
\nabla_{e_{i}} \nabla_{e_{i}} \nabla_{u} v= & \nabla_{e_{i}}\left(R\left(u, e_{i}\right) v\right)+\nabla_{e_{i}}\left(\nabla_{\left[e_{i}, u\right]} v\right)+\nabla_{e_{i}} \nabla_{u} \nabla_{e_{i}} v \\
= & \nabla_{u} \nabla_{e_{i}} \nabla_{e_{i}} v+\nabla_{e_{i}}\left(\nabla_{\left[e_{i}, u\right]} v\right)+\nabla_{\left[e_{i}, u\right]} \nabla_{e_{i}} v \\
& +\nabla_{e_{i}}\left(R\left(u, e_{i}\right) v\right)+R\left(u, e_{i}\right) \nabla_{e_{i}} v \\
& +\nabla_{u} \operatorname{Ric}(v)-\nabla_{u}(\operatorname{Ric}(v)) .
\end{aligned}
$$

Expressing $u$ as $u^{j} e_{j}$, we see that $\left[e_{i}, u\right]=e_{i}\left[u^{j}\right] e_{j}$; hence, one may easily verify that

$$
\begin{aligned}
\nabla_{\left[e_{i}, u\right]} \nabla_{e_{i}} v & =\operatorname{div}[\nabla v \cdot \nabla u], \\
\nabla_{e_{i}}\left(\nabla_{\left[e_{i}, u\right]} v\right) & =\operatorname{div}\left[\nabla v \cdot \nabla u^{t}\right],
\end{aligned}
$$

so that

$$
\begin{aligned}
-\triangle \nabla_{u} v= & -\nabla_{u} \Delta v-\operatorname{div}\left[\nabla v \cdot \nabla u^{t}+\nabla v \cdot \nabla u\right] \\
& -\left(\nabla_{u} \operatorname{Ric}\right) \cdot v-\operatorname{Tr}[\nabla(R(u, \cdot) v)+R(u \cdot) \nabla v] .
\end{aligned}
$$

Using the fact that $\operatorname{div} \nabla_{u} v=\operatorname{Tr}(\nabla u \cdot \nabla v)+\operatorname{Ric}(u, v)$, and combining terms involving the Ricci curvature gives the result. q.e.d.

We remark that if we embed $M$ into its double $\tilde{M}$, smoothly extending $g$, and let $(1-\hat{\mathcal{L}})$ denote the operator $\left(1-2 \operatorname{Def}^{*}\right.$ Def $)$ on $\tilde{M}$, then it makes sense for $R \circ(1-\hat{\mathcal{L}}) \circ E$ to formally act on an arbitrary vector fields on $\bar{M}$. Here, $R$ denotes restriction and $E$ denotes extension; see the proof of Theorem 2 for a more detailed construction of such an operator. It follows that the above lemma also holds for the groups $\mathcal{D}_{\mu, N}^{s}$ and $\mathcal{D}_{\mu, \text { mix }}^{s}$ when the operator $(1-\mathcal{L})$ acting vector fields which vanish on $\partial M$ is replaced by $R \circ(1-\hat{\mathcal{L}}) \circ E$.

\section{Proof of Theorem 2}

Let us denote the covariant material time derivative by $(\nabla / d t)$. For the remainder of this section we shall, for convenience, set $\alpha=1$. The unbounded, self-adjoint operator $(1-\mathcal{L})=\left(1-2\right.$ Def $^{*}$ Def $)$ on $L^{2}(T M)$ has domain $H^{2}(T M) \cap H_{0}^{1}(T M)$.

Proposition 4. For $s>(n / 2)+1$, let $\eta(t)$ be a curve in $\mathcal{D}_{\mu, D}^{s}$, and set $u(t)=\dot{\eta} \circ \eta(t)^{-1}$. Then $u$ is a solution of the initial-boundary 
value problem (1.3) with Dirichlet boundary conditions $u=0$ on $\partial M$ if and only if

$$
\overline{\mathcal{P}}_{\eta} \circ\left[\frac{\nabla \dot{\eta}}{d t}+[\mathcal{U}(u)+\mathcal{R}(u)] \circ \eta\right]=0
$$

where

$$
\begin{gathered}
\mathcal{U}(u)=(1-\mathcal{L})^{-1}\left\{\operatorname{div}\left[\nabla u \cdot \nabla u^{t}+\nabla u \cdot \nabla u-\nabla u^{t} \cdot \nabla u\right]\right\} \\
\mathcal{R}(u)=(1-\mathcal{L})^{-1}\{\operatorname{Tr}[\nabla(R(u, \cdot) u)+R(u, \cdot) \nabla u+R(\nabla u, \cdot) u] \\
\left.-\left(\nabla_{u} \operatorname{Ric}\right) \cdot u+\nabla u^{t} \cdot \operatorname{Ric}(u)\right\},
\end{gathered}
$$

and $\overline{\mathcal{P}}_{\eta}: T_{\eta} \mathcal{D}_{D}^{s} \rightarrow T_{\eta} \mathcal{D}_{\mu, D}^{s}$ is the Stokes projector.

Proof. We first set $\nu=0$. Covariantly differentiating $\dot{\eta}=u \circ \eta$ yields

$$
\frac{\nabla \dot{\eta}}{d t} \circ \eta^{-1}=\partial_{t} u+\nabla_{u} u
$$

Using Lemma 3, we obtain that

$$
\begin{aligned}
(1-\mathcal{L})\left(\frac{\nabla \dot{\eta}}{d t} \circ \eta^{-1}\right)= & \left(1-\triangle_{r}\right) \partial_{t} u+(1-\mathcal{L}) \nabla_{u} u \\
= & \left(1-\triangle_{r}\right) \partial_{t} u+\nabla_{u}\left(1-\triangle_{r}\right) u \\
& -\operatorname{div}\left[\nabla u \cdot \nabla u^{t}+\nabla u \cdot \nabla u\right] \\
& -\operatorname{grad} \operatorname{Tr}(\nabla u \cdot \nabla u)-\operatorname{grad} \operatorname{Ric}(u, u) \\
& -\operatorname{Tr}[\nabla(R(u, \cdot) u)+R(u, \cdot) \nabla u]+\left(\nabla_{u} \operatorname{Ric}\right) \cdot u .
\end{aligned}
$$

Now

$$
\nabla u^{t} \cdot \triangle u=\operatorname{div}\left[\nabla u^{t} \cdot \nabla u\right]+\operatorname{grad} \phi-\operatorname{Tr} R(\nabla u, \cdot) u-\nabla u^{t} \cdot \operatorname{Ric}(u),
$$

for some $\phi: M \rightarrow \mathbb{R} ;$ hence,

$$
\left(1-\triangle_{r}\right) \partial_{t} u+\nabla_{u}\left(1-\triangle_{r}\right) u-\nabla u^{t} \cdot \triangle u=-\operatorname{grad} p
$$

if and only if

$$
\frac{\nabla \dot{\eta}}{d t} \circ \eta^{-1}+\mathcal{U}(u)+\mathcal{R}(u)=-(1-\mathcal{L})^{-1} \operatorname{grad} \tilde{p},
$$

for some $\tilde{p}: M \rightarrow \mathbb{R}$, and by Proposition 1 , this is precisely equation (7.1). q.e.d. 
We can now proceed with the proof of the theorem. We first consider the inviscid case first with the viscosity $\nu=0$.

By Proposition 4, the geodesic flow of the invariant metric $\langle\cdot, \cdot\rangle$ is the solution of

$$
\frac{\nabla \dot{\eta}}{d t}=\overline{\mathcal{S}}_{\eta}(\dot{\eta}):=\left(1-\mathcal{P}_{\eta}\right) \frac{\nabla \dot{\eta}}{d t}-\mathcal{P}_{\eta} \circ\left(\overline{\mathcal{U}}_{\eta}+\overline{\mathcal{R}}_{\eta}\right) \dot{\eta}
$$

where $\overline{\mathcal{S}}$ is the bundle map covering the identity given on each fiber by $\overline{\mathcal{S}}_{\eta}$, and

$\overline{\mathcal{U}}_{\eta}\left(X_{\eta}\right)=\left[\mathcal{U}\left(X_{\eta} \circ \eta^{-1}\right)\right] \circ \eta, \quad \overline{\mathcal{R}}_{\eta}\left(X_{\eta}\right)=\left[\mathcal{R}\left(X_{\eta} \circ \eta^{-1}\right)\right] \circ \eta \forall X_{\eta} \in T_{\eta} \mathfrak{G}_{\mu}^{s}$.

Now the second tangent bundle $T^{2} \mathcal{D}_{\mu, D}^{s}$ is identified with $H^{s}$ maps $\mathcal{Y}: M \rightarrow T^{2} M$ which cover some $X_{\eta} \in T_{\eta} \mathcal{D}_{\mu, D}^{s}$. The second-order vector field $\ddot{\eta}: M \rightarrow T^{2} M$ is just such a map, covering $\dot{\eta} \in T_{\eta} \mathcal{D}_{\mu, D}^{s}$.

Using a local representation, we may express the material time derivative above as the system

$$
\begin{aligned}
\dot{\eta} & =V_{\eta}, \\
\ddot{\eta} & =\frac{d V_{\eta}}{d t}=\mathcal{B}(\eta, \dot{\eta})=-\Gamma_{\eta}(\dot{\eta}, \dot{\eta})+\overline{\mathcal{S}}_{\eta}(\dot{\eta}), \\
\eta(0) & =e \\
V_{\eta}(0) & =u_{0},
\end{aligned}
$$

since $\nabla \dot{\eta} / d t=\ddot{\eta}+\Gamma_{\eta}(\dot{\eta}, \dot{\eta})$, where $\Gamma_{\eta}(\dot{\eta}, \dot{\eta})$ is the Christoffel map, given in a local coordinate chart on $M$ by $\Gamma_{\eta(x)}(\dot{\eta}, \dot{\eta})=\Gamma_{j k}^{i}(x)\left(\dot{\eta} \circ \eta^{-1}\right)^{j}\left(\dot{\eta} \circ \eta^{-1}\right)^{k}$. $\mathcal{B}(\eta, \dot{\eta})$ is the principal part of the geodesic spray of $\langle\cdot, \cdot\rangle$ on $\mathcal{D}_{\mu, D}^{s}$; hence, with $\mathcal{U}$ denoting a local open neighborhood of $\eta \in \mathcal{D}_{\mu, D}^{s}$, to establish the first assertion we shall prove that $\mathcal{B}$ maps $\mathcal{U} \times H_{\eta}^{s}(T M)$ into $H_{\eta}^{s}(T M)$, and that $\mathcal{B}$ is $C^{\infty}$. The result then follows by application of the fundamental theorem of ordinary differential equations on Hilbert manifolds (see [21], Theorem 2.6), and the existing time-reversal symmetry $t \mapsto-t$.

As the Christoffel map is a $C^{\infty}$ map of $\mathcal{U} \times H_{\eta}^{s}(T M)$ into $H_{\eta}^{s}(T M)$ (since $g$ is $C^{\infty}$ and $H^{s}$ is a multiplicative algebra), we must show that $\overline{\mathcal{S}}_{\eta}$ is $C^{\infty}$. Since $\overline{\mathcal{P}}_{\eta}: T_{\eta} \mathcal{D}_{D}^{s} \rightarrow T_{\eta} \mathcal{D}_{\mu, D}^{s}$ is $C^{\infty}$ by Proposition 1 , to show that $\mathcal{P}_{\eta} \circ \overline{\mathcal{U}}_{\eta}: T_{\eta} \mathcal{D}_{\mu, D}^{s} \rightarrow T_{\eta} \mathcal{D}_{\mu, D}^{s}$ is $C^{\infty}$ it suffices to prove that

$$
{\overline{(1-\mathcal{L})^{-1}}}_{\eta} \circ \overline{\operatorname{div}}_{\eta} \circ\left[\nabla\left(\dot{\eta} \circ \eta^{-1}\right) \circ \eta \cdot \nabla\left(\dot{\eta} \circ \eta^{-1}\right) \circ \eta\right]: T_{\eta} \mathcal{D}_{\mu, D}^{s} \rightarrow T_{\eta} \mathcal{D}_{D}^{s}
$$


and

${\overline{(1-\mathcal{L})^{-1}}}_{\eta} \circ \overline{\operatorname{grad}}_{\eta} \circ \operatorname{Tr}\left[\nabla\left(\dot{\eta} \circ \eta^{-1}\right) \circ \eta \cdot \nabla\left(\dot{\eta} \circ \eta^{-1}\right) \circ \eta\right]: T_{\eta} \mathcal{D}_{\mu, D}^{s} \rightarrow T_{\eta} \mathcal{D}_{D}^{s}$

are $C^{\infty}$ bundle maps. But this follows from Lemmas 5 and 6 together with Proposition 5. Since $R$ and Ric are $C^{\infty}$ on $M$, a similar argument shows that $\mathcal{P}_{\eta} \circ \overline{\mathcal{R}}_{\eta}: T_{\eta} \mathcal{D}_{\mu, D}^{s} \rightarrow T_{\eta} \mathcal{D}_{\mu, D}^{s}$ is $C^{\infty}$ as well.

We next prove that $\left(1-\overline{\mathcal{P}}_{\eta}\right) \circ(\nabla \dot{\eta} / d t)$ is $C^{\infty}$. Since $\partial_{t} u \in T_{e} \mathcal{D}_{\mu, D}^{s}$,

$$
\overline{\mathcal{P}}_{\eta} \circ \frac{\nabla \dot{\eta}}{d t}=\left[\partial_{t} u+\mathcal{P}_{e}\left(\nabla_{u} u\right)\right] \circ \eta
$$

so that

$$
\left(1-\overline{\mathcal{P}}_{\eta}\right) \circ(\nabla \dot{\eta} / d t)=-(1-\mathcal{L})^{-1} \operatorname{grad} p \circ \eta,
$$

where $p$ depends on $v$ and the pair $(v, p)$ is a solution of the Stokes problem

$$
\begin{gathered}
\left(1-\triangle_{r}\right) v+\operatorname{grad} p=(1-\mathcal{L}) \nabla_{u} u \\
\quad \operatorname{div} v=0 \\
v=0 \text { on } \partial M
\end{gathered}
$$

Since $s>(n / 2)+1,(1-\mathcal{L}) \nabla_{u} u$ is in $H^{s-3}(T M)$; the argument in Step 3 of the proof of Theorem 2 then gives a unique solution $(v, p) \in$ $\mathcal{V}_{\mu}^{s-1} \times H^{s-2}(M) / \mathbb{R}$. If $-1<s-3<0$, then the pair $(v, p)$ is interpreted as a weak solution.

A priori, $(1-\mathcal{L})^{-1} \operatorname{grad} p$ is only in $H^{s-1}$, but we shall show that, in fact, $(1-\mathcal{L})^{-1} \operatorname{grad} p$ is actually of class $H^{s}$. We have that

$$
(1-\mathcal{L})^{-1} \operatorname{grad} p=(1-\mathcal{L})^{-1} \operatorname{grad} \triangle^{-1} \operatorname{div}(1-\mathcal{L})\left(v-\nabla_{u} u\right) .
$$

We embed $M$ into its double $\tilde{M}$, extending $g$ to $\tilde{M}$, and choose a $C^{\infty}$ extension of $u$ to $\tilde{M}$. For any vector bundle $\mathcal{E}$ over $M$, let

$$
E: H^{s}(\mathcal{E} \downarrow M) \rightarrow H^{s}(\mathcal{E} \downarrow \tilde{M}),\left.\quad E(\xi)\right|_{M}=\xi
$$

denote the linear extension operator, and let $R$ denote the corresponding restriction operator. Let $\hat{\mathcal{L}}$ denote $R \circ \mathcal{L} \circ E$; then it makes sense to form the commutator of the operators div with $\hat{\mathcal{L}}$, and the operator

$$
[\operatorname{div}, \hat{\mathcal{L}}]: H^{r}(T M) \rightarrow H^{r-2}(T M)
$$


is continuous. Notice that as $\mathcal{L}$ is a local operator, if $w=0$ on $M$, then $\hat{\mathcal{L}} w=0$ by the property of the extension operator given above. Since $\operatorname{div} v=0$,

$$
-(1-\mathcal{L})^{-1} \operatorname{grad} \triangle^{-1} \operatorname{div} \mathcal{L} v=-(1-\mathcal{L})^{-1} \operatorname{grad} \triangle^{-1}[\operatorname{div}, \hat{\mathcal{L}}] v,
$$

which is in $H^{s}(T M) \cap H_{0}^{1}(T M)$, since

$$
\begin{array}{r}
-(1-\mathcal{L})^{-1} \operatorname{grad} \triangle^{-1}[\operatorname{div}, \hat{\mathcal{L}}]: \\
\rightarrow H^{s-1}(T M) \cap H_{0}^{1}(T M) \\
\rightarrow H^{s}(T M) \cap H_{0}^{1}(T M)
\end{array}
$$

is a compact operator.

The identical argument shows that $-(1-\mathcal{L})^{-1} \operatorname{grad} \triangle^{-1}[\operatorname{div}, \hat{\mathcal{L}}] \nabla_{u} u$ is in $H^{s}(T M) \cap H_{0}^{1}(T M)$, since $\nabla_{u} u$ is in $H^{s-1}(T M) \cap H_{0}^{1}(T M)$. Since $\operatorname{div} \nabla_{u} u=\operatorname{Tr}(\nabla u \cdot \nabla u)+\operatorname{Ric}(u, u)$ is an $H^{s-1}$ vector field on $M$, and since

$$
-(1-\mathcal{L})^{-1} \operatorname{grad} \triangle^{-1} \hat{\mathcal{L}}: H^{s-1}(T M) \rightarrow H^{s}(T M) \cap H_{0}^{1}(T M)
$$

compactly, we see that

$$
-(1-\mathcal{L})^{-1} \operatorname{grad} \triangle^{-1} \operatorname{div} \mathcal{L} \nabla_{u} u
$$

is in fact of class $H^{s}$. Regularity up to the boundary immediately follows from the fact that $\nabla_{u} u=0$ on $\partial M$. Thus $(1-\mathcal{L})^{-1} \operatorname{grad} p$ is in $H^{s}$, and from Section 10, it follows that $\left[(1-\mathcal{L})^{-1} \operatorname{grad} p\right] \circ \eta$ is in $H_{\eta}^{s}(T M)$.

The fact that $u$ is the unique solution of (1.3) is the statement of Proposition 4. That $u$ is in $C^{0}\left(I, \mathcal{V}_{\mu}^{s}\right) \cap C^{1}\left(I, \mathcal{V}_{\mu}^{s-1}\right)$ and depends continuously on the initial data $u_{0}$ follows from the fact that the inversion map $\left(\eta \mapsto \eta^{-1}\right): \mathcal{D}^{s} \rightarrow \mathcal{D}^{s}$ is only $C^{0}$, but is $C^{1}$ when considered as a map from $\mathcal{D}^{s}$ into $\mathcal{D}^{s-1}$. This proves the theorem.

\section{Proof of Theorem 3}

Let $A=-P \triangle_{r}$ denote the Stokes operator. The operator $A^{-1}$ is continuous from $H=\left\{u \in L^{2}(T M) \mid \operatorname{div} u=0, g(u, n)=0\right.$ on $\left.\partial M\right\}$ into $D(A)=H^{2}(T M) \cap V$, where $V=\left\{u \in H_{0}^{1}(T M) \mid \operatorname{div} u=0\right\}$. The embedding of $V$ in $H$ is compact; thus $A^{-1}$ is a self-adjoint continuous compact operator in $H$, so that there exist eigenvalues

$$
0<\lambda_{1} \leq \lambda_{1}, \ldots, \quad \lambda_{j} \rightarrow \infty
$$


and eigenfunctions $w_{j} \in D(A)$ for $j \in \mathbb{N}$, such that

$$
A w_{j}=\lambda_{j} w_{j}, \quad j \in \mathbb{N} .
$$

Let $P_{j}: H_{0}^{1} \rightarrow V_{j}:=\operatorname{span}\left\{w_{1}, \ldots, w_{j}\right\}$ denote the projection onto the span of the first $j$ eigenfunctions of $A$.

Our approximating equation will be

$$
\partial_{t} u_{j}+P_{j}\left(\nabla_{u_{j}} u_{j}+\mathcal{U}^{\alpha}\left(u_{j}\right)\right)=0, \quad u_{j}(0)=P_{j} u_{0} .
$$

By Lemma 3, it follows that

$$
\left\langle P_{j}\left(\nabla_{u_{j}} u_{j}+\mathcal{U}^{\alpha}\left(u_{j}\right)\right), u_{j}\right\rangle=0
$$

so that computing the $\langle\cdot, \cdot\rangle_{e}$ inner-product of (8.1) with $u_{j}$ shows that

$$
\begin{aligned}
\frac{d}{d t}\left(\left|u_{j}\right|_{0}^{2}+\alpha^{2}\left|A^{\frac{1}{2}} u_{j}\right|_{0}^{2}\right) & \leq-\nu\left(\left|A^{\frac{1}{2}} u_{j}\right|_{0}^{2}+\alpha^{2}\left|A u_{j}\right|_{0}^{2}\right) \\
& \leq\left|P_{j} u_{0}\right|_{0}^{2} .
\end{aligned}
$$

Thus, it follows that (8.1) is solvable for all $t \geq 0$ whenever $\nu \geq 0$, for each $j$. Next, we estimate higher-order derivatives of $u_{j}$, so that we can pass to the limit as $j \rightarrow \infty$. We only consider the range of $s \in\left(\frac{n}{2}+1,3\right)$ for $n=2,3$.

Using the Moser or calculus inequalities

$$
|f \cdot g|_{s} \leq C\left(|f|_{s}|g|_{L^{\infty}}+|f|_{L^{\infty}}|g|_{s}\right) \quad f, g \in H^{s}(M), s \geq \frac{n}{2}+1,
$$

we obtain the basic estimate

$$
\frac{d}{d t}\left|u_{j}(t)\right|_{s}^{2} \leq C\left|u_{j}\right|_{C^{1}}\left|u_{j}\right|_{s}^{2}-\nu\left\langle A^{\frac{s}{2}} A u_{j}, A^{\frac{s}{2}} u_{j}\right\rangle_{0} .
$$

Since $A u_{j} \in D\left(A^{\frac{s}{2}}\right)$ for $s \in\left(\frac{n}{2}+1,3\right)$, the definition of $D\left(A^{\frac{s}{2}}\right)$, as given by [39] for example, shows that

$$
-\nu\left\langle A^{\frac{s}{2}} A u_{j}, A^{\frac{s}{2}} u_{j}\right\rangle_{0}=-\nu \sum_{j=1}^{\infty} \lambda^{s+1}\left|u_{j}\right|_{0}^{2} \leq 0,
$$

so that $y^{\prime}(t) \leq a(t) y(t)$, where $y(t)=\left|u_{j}(t)\right|_{s}^{2}$ and $a(t)=C\left|u_{j}(t)\right|_{C^{1}}$. Hence $y(t) \leq e^{\int_{0}^{t} a(s) d s} y_{0}$. With this a priori bound, standard compactness arguments (see, for example, the proof of Theorem 1.2 in Chapter 16 of [38]) allow us to pass to the limit as $j \rightarrow \infty$ and obtain the existence of unique $u \in L^{\infty}\left([0, T), T_{e} \mathcal{D}_{\mu, D}^{s}\right)$, where $T$ is independent of $\nu>0$. This proves the theorem. 


\section{Proof of Theorem 4}

The existence of the unique Levi-Civita covariant derivative of the right invariant metric $\langle\cdot, \cdot\rangle$ on $\mathcal{D}_{\mu, D}^{s}$ is an immediate consequence of the smoothness of the geodesic flow of $\langle\cdot, \cdot\rangle$ provided by Theorem 2 . The formulas for $\tilde{\nabla}$ then follow from the fundamental theorem of Riemannian geometry.

As to the properties of the curvature operator, right invariance of $\tilde{R}$ follows from the right invariance of $\tilde{\nabla}$. Next we prove that $\tilde{R}$ is bounded in $H^{s}$ for $s>\frac{n}{2}+2$.

Extend $X_{\eta}, Y_{\eta}, Z_{\eta} \in T_{\eta} \mathcal{D}_{\mu, D}^{s}$ to smooth right invariant vector fields $x^{r}, y^{r}, z^{r}$ on $\mathcal{D}_{\mu, D}^{s}$ and let $x=x^{r}(e), y=y^{r}(e)$, and $z=z^{r}(e)$. Let

$$
M_{x} y=\left(1-\mathcal{P}_{e}\right) \circ \nabla_{x} y+(1 / 2) \mathcal{P}_{e} \circ(\mathfrak{U}(x, y)+\mathfrak{R}(x, y)) .
$$

As the proof of Theorem 2 shows, $M$ has the following property:

If $x$ and $y$ are $H^{s}$ divergence-free vector fields on $M$, and $s$ is sufficiently large so that $H^{s-1}(T M)$ forms a multiplicative algebra, then there exists a positive constant $c$, such that $\left|M_{x} y\right|_{s} \leq$ $c|x|_{s}|y|_{s}$.

Now, since $\tilde{\nabla}$ is right invariant, we have that

$$
\begin{aligned}
\tilde{R}_{\eta}\left(X_{\eta}, Y_{\eta}\right) z_{\eta}^{r}= & \left(\tilde{\nabla}_{y^{r}} \tilde{\nabla}_{x^{r}} z^{r}\right)_{\eta}-\left(\tilde{\nabla}_{x^{r}} \tilde{\nabla}_{y^{r}} z^{r}\right)_{\eta}+\left(\tilde{\nabla}_{\left[x^{r}, y^{r}\right]} z^{r}\right)_{\eta} \\
= & {\left[\left(\nabla_{y}+M_{y}\right)\left(\nabla_{x}+M_{x}\right) z\right] \circ \eta } \\
& -\left[\left(\nabla_{x}+M_{x}\right)\left(\nabla_{y}+M_{y}\right) z\right] \circ \eta \\
& +\left[\left(\nabla_{[x, y]}+M_{[x, y]}\right) z\right] \circ \eta \\
= & {\left[\left(\nabla_{y} \nabla_{x}-\nabla_{x} \nabla_{y}+\nabla_{[x, y]}\right) z\right] \circ \eta } \\
& +\left[\left(M_{y} M_{x}-M_{x} M_{y}+M_{[x, y]}\right) z\right] \circ \eta \\
& +\left[\left\{\nabla_{x}, M_{y}\right\} z+\left\{M_{x}, \nabla_{y}\right\} z\right] \circ \eta,
\end{aligned}
$$

where $\{\cdot, \cdot\}$ denotes the commutator of operators.

Since $R(x, y) z \circ \eta=\left[\left(D_{y} D_{x}-D_{x} D_{y}+D_{[x, y]}\right) z\right] \circ \eta$, this term is clearly continuous in $H^{s}$, as $R$, the curvature of $\nabla$ on $M$, is $C^{\infty}$.

That $(x, y, z) \mapsto\left[\left(M_{y} M_{x}-M_{x} M_{y}+M_{[x, y]}\right) z\right] \circ \eta$ is continuous in $H^{s}$ follows from the above property of $M$; namely, $[x, y] \in H^{s-1}(T M)$ and for $s>(n / 2)+2, H^{s-2}(T M)$ forms a multiplicative algebra so that

$$
\left|M_{[x, y]} z\right|_{s-1} \leq c|[x, y]|_{s-1}|z|_{s-1} \leq c|x|_{s}|y|_{s}|z|_{s} .
$$


Finally, continuity of $(x, y, z) \mapsto\left[\left\{\nabla_{x}, M_{y}\right\} z+\left\{M_{x}, \nabla_{y}\right\} z\right] \circ \eta$ in $H^{s}$ follows from the fact that the commutator terms are both order-zero differential operators, together with the property of the multiplicative algebra.

\section{Smoothness of differential bundle maps over the identity}

Let $\mathfrak{G}^{s}$ denote either $\mathcal{D}_{D}^{s}, \mathcal{D}_{N}^{s}$, or $\mathcal{D}_{\text {mix }}^{s}$. Suppose $L: H^{s}(E) \rightarrow$ $H^{s-l}(F)$ is an order $l$ differential operator between sections of two vector bundles $E$ and $F$ over $M$. The purpose of this appendix is to carefully explain why $R_{\eta} \circ L \circ R_{\eta^{-1}}: H^{s}(M, E) \downarrow \mathfrak{G}^{s} \rightarrow H^{s-l}(M, F) \downarrow \mathfrak{G}^{s}$ is smooth, even though the map $\eta \mapsto \eta^{-1}: \mathfrak{G}^{s} \longrightarrow \mathfrak{G}^{s}$ is only $C^{0}$. That $R_{\eta} \circ L \circ R_{\eta^{-1}}$ is $C^{\infty}$ follows from the special structure of exact sequences covering the identity map.

A sequence of vector bundle maps over the identity $E \stackrel{f}{\rightarrow} F \stackrel{g}{\rightarrow} G$ is exact at $F$ if range $(f)=\operatorname{ker}(g)$; split fiber exact if $\operatorname{ker}(f)$, range $(f)=\operatorname{ker}(g)$, and range $(g)$ split in $E, F$, and $G$, respectively; and bundle exact if additionally $\operatorname{ker}(f)$, range $(f)=\operatorname{ker}(g)$, and $\operatorname{range}(g)$ are subbundles. It is standard ([2], Proposition 3.4.20) that a split fiber exact sequence is bundle exact, so that if $E, F$, and $G$ are Hilbert vector bundles, and the sequence is exact at $F$, then $\operatorname{ker}(f), \operatorname{range}(f)=\operatorname{ker}(g)$, and range $(g)$ are subbundles.

Let $\tilde{M}$ denote the double of $M$, and set $H^{s}\left(\Lambda^{k}\right)=H^{s}\left(\Lambda^{k}(\tilde{M})\right)$, the $H^{s}$ class sections of $\Lambda^{k}(\tilde{M})$. Let $H_{\eta}^{s}\left(\Lambda^{k}\right)$ denote the $H^{s}$ class maps of $\tilde{M}$ into $\Lambda^{k}(\tilde{M})$ which cover $\eta$.

Lemma 4. For $s>(n / 2)+1$, the map

$$
(\eta \mapsto T \eta): \mathcal{D}^{s} \rightarrow\left[H^{s}(T M)^{*} \otimes H_{\eta}^{s-1}(T M)\right] \downarrow \mathcal{D}^{s}
$$

is $C^{\infty}$.

Proof. For each $x \in M$, the metric $g$ induces a natural inner-product, say $\bar{g}$, on elements of $T_{x}^{*} M \otimes T_{\eta(x)} M$, and hence a weak $L^{2}$ metric on $H^{s}(T M)^{*} \otimes H_{\eta}^{s-1}(T M)$ given by $\int_{M} \bar{g}(\cdot, \cdot) \mu$. There exists a unique LeviCivita covariant derivative associated with this weak $L^{2}$ metric which we denote by $\bar{\nabla}$. The covariant derivative $\bar{\nabla}$ is induced by the connector $\mathcal{K}$ which is the functorial lift of the connector $K$ uniquely associated with the metric $\bar{g}$ thru the fundamental theorem of Riemannian geometry (see Theorem 9.1 in [13]). 
Let us denote the map $\eta \mapsto T \eta$ by $s$, i.e., $s(\eta)=T \eta$. Continuity of $s$ is immediate. Thus, we shall show that $s$ is of class $C^{1}$. Let $\epsilon \mapsto \eta^{\epsilon}$ be a smooth curve in $\mathcal{D}^{s}$ such that $\eta^{0}=\eta$ and $\left.(d / d \epsilon)\right|_{\epsilon=0} \eta^{\epsilon}=V_{\eta} \in T_{\eta} \mathcal{D}^{s}$; then, $\bar{\nabla}_{V_{\eta}} s(\eta) \in H^{s}(T M)^{*} \otimes H_{\eta}^{s-1}(T M)$ is computed as

$$
\bar{\nabla}_{V_{\eta}} s(\eta)=\left.\frac{d}{d \epsilon}\right|_{\epsilon=0} s\left(\eta^{\epsilon}\right)=\left.\frac{d}{d \epsilon}\right|_{\epsilon=0} T \eta^{\epsilon}=\nabla V_{\eta}
$$

where $\nabla$ denotes the unique Levi-Civita covariant derivative in the pullback bundle $\eta^{*}(T M)$ associated to the metric $g$ on $M$. Specifically, for $W \in T_{x} M$ and $V_{\eta} \in \eta^{*}(T M), \nabla_{W} V_{\eta}(x)$ has the local expression

$$
\nabla_{W} V_{\eta}(x)=T V_{\eta}(x) \cdot(T \eta(x) \cdot W(x))+\Gamma_{\eta(x)}\left(V_{\eta}(x), T \eta(x) \cdot W(x)\right),
$$

where $\Gamma_{\eta(x)}$ denotes the Christoffel symbol of the metric $g$ evaluated at the point $\eta(x) \in M$.

We compute the operator norm of

$$
\bar{\nabla} s(\eta) \in \operatorname{Hom}\left(H_{\eta}^{s}(T M), H^{s}(T M)^{*} \otimes H_{\eta}^{s-1}(T M)\right)
$$

which we shall denote by $|\cdot|_{\text {op }}$. We have that

$$
\begin{aligned}
|\bar{\nabla} s(\eta)|_{\text {op }} & =\sup _{V_{\eta} \in H_{\eta}^{s},\left|V_{\eta}\right|_{s}=1}\left|\nabla V_{\eta}\right|_{H^{s}(T M)^{*} \otimes H_{\eta}^{s-1}(T M)} \\
& =\sup _{V_{\eta} \in H_{\eta}^{s},\left|V_{\eta}\right|_{s}=1} \sup _{W \in H^{s},|W|_{s}=1}\left|\nabla_{W} V_{\eta}\right|_{s-1} \\
& \leq \sup _{V_{\eta} \in H_{\eta}^{s},\left|V_{\eta}\right|_{s}=1} \sup _{W \in H^{s},|W|_{s}=1}|\nabla V|_{s-1}|W|_{s} \\
& <C\left(g,|T \eta|_{s-1}\right)<\infty .
\end{aligned}
$$

Computing the supremum of $|\bar{\nabla} s(\eta)|_{\text {op }}$ in a neighborhood of $\eta$ yields the $C^{1}$ topology; as the supremum is finite, we have established that $s$ is a $C^{1}$ map.

To see that $s$ is of class $C^{2}$, we compute in a local chart

$$
\begin{aligned}
\left.\frac{d}{d \epsilon}\right|_{\epsilon=0} \nabla V_{\eta^{\epsilon}}= & T V_{\eta}(x) \cdot \nabla_{W} V_{\eta}(x) \\
& +T \Gamma_{\eta(x)} \cdot T \eta(x)\left(V_{\eta}(x), T \eta(x) \cdot W(x)\right) \\
& +\Gamma_{\eta(x)}\left(V_{\eta}(x), \nabla_{W} V_{\eta}(x)\right) .
\end{aligned}
$$

Since $T \eta$ is in the multiplicative algebra $H^{s-1}$, and $\Gamma \in C^{\infty}$, the same argument as above shows that $s$ is $C^{2}$. In particular, we see that the $k$ th 
derivative of $s$ is a rational combination of $\eta, T \eta, \nabla V_{\eta}$ and derivatives of $\Gamma$, which combined with our argument showing that $s$ is $C^{1}$ together with the fact that multiplication of $H^{s-1}$ maps is smooth, shows that $s$ is $C^{k}$ for any integer $k \geq 0$, and hence that $s$ is $C^{\infty}$. q.e.d.

Define $\bar{d}: H_{\eta}^{s}\left(\Lambda^{k}\right) \downarrow \mathcal{G}^{s} \rightarrow H_{\eta}^{s-1}\left(\Lambda^{k+1}\right) \downarrow \mathfrak{G}^{s}$ to be the bundle map covering the identity given by

$$
\bar{d}_{\eta}\left(\alpha_{\eta}\right)=\left[d\left(\alpha_{\eta} \circ \eta^{-1}\right)\right] \circ \eta \quad \forall \alpha_{\eta} \in H_{\eta}^{s}\left(\Lambda^{k}\right) .
$$

Similarly, define

$$
\bar{\delta}: H_{\eta}^{s}\left(\Lambda^{k}\right) \downarrow \mathcal{G}^{s} \rightarrow H_{\eta}^{s-1}\left(\Lambda^{k-1}\right) \downarrow \mathfrak{G}^{s}
$$

by $\bar{\delta}_{\eta}=\left[\delta\left(\alpha_{\eta} \circ \eta^{-1}\right] \circ \eta\right.$. Lemma A.2 of [13] states that these bundle maps are smooth. We give the following proof. First note that, as $d$ is an antiderivation satisfying

$$
d(\alpha \wedge \beta)=d \alpha \wedge \beta+(-1)^{k} \alpha \wedge d \beta \quad \forall \alpha \in \Lambda^{k},
$$

it suffices to give the proof for $k=1$, in which case $d \alpha=\nabla \alpha-(\nabla \alpha)^{t}$, where $\nabla$ is the Levi-Civita covariant derivative on $T^{*} M$. Using the chain rule, we see that $\bar{d}_{\eta}=\left[\nabla \circ T \eta^{-1}-\left(\nabla \circ T \eta^{-1}\right)^{t}\right] \circ \eta$. Now $T \eta^{-1}$ is of class $H^{s-1}$ whenever $\eta$ is an $H^{s}$ class diffeomorphism, so the proof of Lemma 4 shows that $\bar{d}$ is $C^{\infty}$. The fact that $\bar{\delta}$ is $C^{\infty}$ follows from a similar argument. We also have the following

Lemma 5. For $s>(n / 2)+1$, if $X_{\eta}, Y_{\eta} \in H_{\eta}^{s}(T \tilde{M})$, then

$$
\overline{\operatorname{div}}_{\eta} \circ\left[\nabla\left(X_{\eta} \circ \eta^{-1}\right) \circ \eta \cdot \nabla\left(Y_{\eta} \circ \eta^{-1}\right) \circ \eta\right] \in H_{\eta}^{s-2}(T \tilde{M}) .
$$

Proof. We identify $X_{\eta}, Y_{\eta} \in H_{\eta}^{s}(T \tilde{M})$ with $\alpha_{\eta}, \beta_{\eta} \in H_{\eta}^{s}\left(\Lambda^{1}\right)$, respectively. It then suffices to prove that $\bar{\delta}_{\eta} \circ\left(\bar{d}_{\eta}\left(\alpha_{\eta}\right) \cdot \bar{d}_{\eta}\left(\beta_{\eta}\right)\right)$ is in $H_{\eta}^{s-2}\left(\Lambda^{1}\right)$, and hence that $\bar{d}_{\eta}\left(\alpha_{\eta}\right) \cdot \bar{d}_{\eta}\left(\beta_{\eta}\right)$ is in $H_{\eta}^{s-1}\left(\Lambda^{1}\right)$ (since $\bar{\delta}$ is $C^{\infty}$ ). But this follows since $H^{s-1}$ is a multiplicative algebra, and $\bar{d}$ is a $C^{\infty}$ bundle map. q.e.d.

A similar argument yields

Lemma 6. For $s>(n / 2)+1$, if $X_{\eta}, Y_{\eta} \in H_{\eta}^{s}(T \tilde{M})$, then

$$
\overline{\operatorname{grad}}_{\eta} \circ \operatorname{Tr}\left[\nabla\left(X_{\eta} \circ \eta^{-1}\right) \circ \eta \cdot \nabla\left(Y_{\eta} \circ \eta^{-1}\right) \circ \eta\right] \in H_{\eta}^{s-2}(T \tilde{M}) .
$$

We shall need Lemma A.3 in [13] which we state as follows: 
Lemma 7. Let $\pi: E \rightarrow M$ be a vector bundle, let $\mathcal{J}$ be a finite dimensional subspace of $H^{s}(E)$ consisting of $C^{\infty}$ elements, and let $\mathfrak{P}: H^{s}(E) \rightarrow \mathcal{J}$ be a continuous orthogonal projector onto $\mathcal{J}$. Then $\overline{\mathcal{J}}=\mathcal{J}_{\eta} \downarrow \mathcal{D}^{s}$ is a subbundle of $H_{\eta}^{r}(M, E) \downarrow \mathcal{D}^{s}$ for $r \leq s$, where $\mathcal{J}_{\eta}=\left\{f \in H^{r}(M, E) \mid f \in R_{\eta} \mathcal{J}\right\}$. Furthermore, $\overline{\mathfrak{P}}: H_{\eta}^{r} \downarrow \mathcal{D}^{s} \rightarrow \overline{\mathcal{J}}$, given by $\overline{\mathfrak{P}}_{\eta}=R_{\eta} \circ \mathfrak{P} \circ R_{\eta^{-1}}$ is a $C^{\infty}$ bundle map.

For the remainder of this appendix, $\bar{A}$ shall denote the bundle map given by $\bar{A}_{\eta}\left(\alpha_{\eta}\right)=\left[A\left(\alpha_{\eta} \circ \eta^{-1}\right)\right] \circ \eta$ for any linear operator $A$ acting on $H^{s}\left(\Lambda^{k}\right)$. We shall use the notation $\overline{\mathcal{W}}$ to denote the bundle $\mathcal{W}_{\eta} \downarrow \mathcal{D}^{s}$ for any vector space $\mathcal{W}$. For example, $\overline{H^{s}\left(\Lambda^{k}\right)}$ shall denote $H_{\eta}^{s}\left(\Lambda^{k}\right) \downarrow \mathcal{D}^{s}$.

Again, for $r \geq 1$, let $\mathcal{V}^{r}$ denote the $H^{r}$ vector fields on $M$ which satisfy the boundary conditions prescribed to elements of $T_{e} \mathfrak{G}^{s}$, and let $\mathcal{V}_{\eta}^{r}=\left\{u \circ \eta: u \in \mathcal{V}^{r}\right\}$.

Proposition 5. Let $\mathcal{L}=-2 \operatorname{Def}^{*}$ Def and define $\overline{\mathcal{L}}$ by

$$
\overline{\mathcal{L}}_{\eta}=T R_{\eta} \circ \mathcal{L} \circ T R_{\eta^{-1}}
$$

Then, for $s>(n / 2)+1$, the bundle maps

$$
\begin{gathered}
\overline{(1-\mathcal{L})}: \mathcal{V}_{\eta}^{s} \downarrow \mathfrak{G}^{s} \rightarrow H_{\eta}^{s-2}(T M) \downarrow \mathfrak{G}^{s}, \\
\overline{(1-\mathcal{L})^{-1}}: H_{\eta}^{s-2}(T M) \downarrow \mathfrak{G}^{s} \rightarrow \mathcal{V}_{\eta}^{s} \downarrow \mathfrak{G}^{s}
\end{gathered}
$$

are $C^{\infty}$.

Proof. By the $L^{2}$ orthogonal Hodge decomposition,

$$
H^{s}\left(\Lambda^{k}\right)=d\left(H^{s+1}\left(\Lambda^{k-1}\right)\right) \oplus \delta\left(H^{s+1}\left(\Lambda^{k+1}\right)\right) \oplus \mathcal{H}_{\text {fields }}^{s, k},
$$

where $\mathcal{H}_{\text {fields }}^{s, k}=\left\{\alpha \in H^{s}\left(\Lambda^{k}\right) \mid d \alpha=0\right.$ and $\left.\delta \alpha=0\right\}$ denotes the Harmonic fields.

Hence,

$$
[\operatorname{ker}(d)]^{\perp}=\delta\left(H^{s+1}\left(\Lambda^{k+1}\right)\right) \text { and }[\operatorname{ker}(\delta)]^{\perp}=d\left(H^{s+1}\left(\Lambda^{k-1}\right)\right) .
$$

Let $\pi$ denote the $L^{2}$ orthogonal projection of $H^{s-1}\left(\Lambda^{k+1}\right)$ onto $\mathcal{H}_{\text {fields }}^{s-1, k+1}$, and let $\mathfrak{p}=\left.\pi\right|_{d\left(H^{s}\left(\Lambda^{k}\right)\right)}$ denote the restriction of $\pi$ to $d\left(H^{s}\left(\Lambda^{k}\right)\right)$, so $\mathfrak{p}: d\left(H^{s}\left(\Lambda^{k}\right)\right) \rightarrow \mathcal{H}_{\text {fields }}^{s-1, k+1}$. Since $\mathcal{H}_{\text {fields }}^{s-1, k+1}$ is a finite dimensional subspace of $H^{s-1}\left(\Lambda^{k+1}\right)$ consisting of $C^{\infty}$ elements, Lemma 7 asserts that 
$\overline{\mathfrak{p}}$ is a smooth bundle map, and that $\operatorname{im}(\overline{\mathfrak{p}})$ and hence $\operatorname{im}(1-\overline{\mathfrak{p}})$ is a subbundle. We may thus form the following exact sequence

$$
H_{\eta}^{s}\left(\Lambda^{k}\right) \downarrow \mathcal{D}^{s} \stackrel{\bar{d}}{\rightarrow} \operatorname{im}(1-\overline{\mathfrak{p}}) \stackrel{\bar{d}}{\rightarrow} H_{\eta}^{s-2}\left(\Lambda^{k+2}\right) \downarrow \mathcal{D}^{s}
$$

Since $\bar{d}$ is a $C^{\infty}$ bundle map, this shows that $\operatorname{ker}(\bar{d})$ and $\operatorname{im}(\bar{d})$ are subbundles. $^{2}$

Now let

$$
\mathfrak{p}_{2}: \delta\left(H^{s}\left(\Lambda^{k}\right)\right) \subset H^{s-1}\left(\Lambda^{k-1}\right) \rightarrow \mathcal{H}_{\text {fields }}^{s-1, k+1}
$$

be the restricted orthogonal projector. Then by the same argument $\overline{\mathfrak{p}}_{2}$ is a smooth bundle map and $\operatorname{im}\left(1-\overline{\mathfrak{p}}_{2}\right)$ is a subbundle. Hence, we may form the exact sequence

$$
H_{\eta}^{s}\left(\Lambda^{k}\right) \downarrow \mathcal{D}^{s} \stackrel{\bar{\delta}}{\rightarrow} \operatorname{im}\left(1-\overline{\mathfrak{p}}_{2}\right) \stackrel{\bar{\delta}}{\rightarrow} H_{\eta}^{s-2}\left(\Lambda^{k-2}\right) \downarrow \mathcal{D}^{s},
$$

and thus obtain that $\operatorname{ker}(\bar{\delta})$ and $\operatorname{im}(\bar{\delta})$ are subbundles.

Using (10.1), we may restrict the domain and range to ensure that the maps $d: \delta\left(H^{s+1}\left(\Lambda^{k+1}\right)\right) \rightarrow d\left(H^{s}\left(\Lambda^{k}\right)\right)$ and $\delta: d\left(H^{s+1}\left(\Lambda^{k-1}\right)\right) \rightarrow$ $\delta\left(H^{s}\left(\Lambda^{k}\right)\right)$ are isomorphisms.

To find the inverse of $d$ between these vector spaces, first let $\omega=\delta \beta$. Then

$$
d \omega=d \delta \beta \Longrightarrow \delta d \omega=\delta d(\delta \beta)=(d \delta+\delta d)(\delta \beta)=-\triangle \delta \beta=-\triangle \omega ;
$$

therefore, $\omega=(-\triangle)^{-1} \delta d \omega=\delta(-\triangle)^{-1} d \omega$, so that $\delta(-\triangle)^{-1}$ is the inverse of $d$. Similarly, we find that $d(-\triangle)^{-1}$ is the inverse of $\delta$.

Next, let

$$
\mathfrak{p}_{3}: \operatorname{ker} \delta=\delta\left(H^{s+1}\left(\Lambda^{k+1}\right)\right) \oplus \mathcal{H}_{\text {fields }}^{s, k} \rightarrow \mathcal{H}_{\text {fields }}^{s, k}
$$

so

$$
\left(1-\mathfrak{p}_{3}\right): \operatorname{ker} \delta \rightarrow \delta\left(H^{s+1}\left(\Lambda^{k+1}\right)\right) .
$$

Now $\overline{\mathfrak{p}}_{3}$ is a smooth bundle map by Lemma 7 , and since $\operatorname{ker}(\delta)$ is a subbundle, we may form the exact sequence

$$
\overline{\operatorname{ker}(\delta)} \stackrel{\overline{\mathfrak{p}}_{3}}{\rightarrow} \overline{\mathcal{H}_{\text {fields }}^{s, k}} \stackrel{0}{\rightarrow} 0 .
$$

\footnotetext{
${ }^{2}$ That $\operatorname{ker}(d)$ and $\operatorname{im}(d)$ are subbundles is the statement of Lemma A.4 in [13]; we have supplied a short proof simply to correct some typographical errors and provide some needed clarification.
} 
Thus, the $\operatorname{im}\left(\overline{\mathfrak{p}}_{3}\right)$ is a subbundle from which it follows that

$$
\left.\operatorname{im}\left(1-\overline{\mathfrak{p}}_{3}\right)=\overline{\delta\left(H^{s+1}\left(\Lambda^{k+1}\right)\right.}\right)
$$

is a subbundle, so that it makes sense to define

$$
\bar{d}: \overline{\delta\left(H^{s+1}\left(\Lambda^{k+1}\right)\right)} \rightarrow \operatorname{im}(\bar{d})
$$

as a smooth bundle isomorphism. A similar argument allows us to define

$$
\bar{\delta}: \overline{d\left(H^{s+1}\left(\Lambda^{k-1}\right)\right)} \rightarrow \operatorname{im}(\bar{\delta})
$$

as smooth bundle isomorphism.

We have shown that the bundle map $\overline{\delta(-\triangle)^{-1}}$ covering the identity is the inverse of $\bar{d}$ which is smooth; hence, by the inverse function theorem, the bundle map $\overline{\delta(-\triangle)^{-1}}$ is also smooth. On the other hand, $\overline{d(-\triangle)^{-1}}$ is the inverse of $\bar{\delta}$, and by the same argument is smooth. Since $\bar{d}$ and $\bar{\delta}$ are $C^{\infty}$, then $\overline{(-\triangle)^{-1}}$ is $C^{\infty}$ on $\operatorname{im}(\bar{d}) \oplus \operatorname{im}(\bar{\delta})$, and hence $\overline{-\triangle}$ is $C^{\infty}$ on ${\overline{\mathcal{H}_{\text {fields }}^{s, k}}}^{\perp}$ again by the inverse function theorem.

Thus far, we have been working with sections of differential $k$-forms over the boundaryless manifold $\tilde{M}$. We shall now restrict our attention to $H^{s}$ class sections of $\Lambda^{1}(\bar{M})$. Letting $n$ denote the outward-pointing normal vector field on $\partial M$, for $r \geq 2$, we define the closed subspace of $H^{r}\left(\Lambda^{1}(M)\right)$ by

$$
\begin{gathered}
H_{A}^{r}=\left\{\alpha \in H^{r}(\Lambda(M)) \mid n\right\lrcorner \alpha=0,\left(\nabla_{n} \alpha^{b}\right)^{\tan }+S_{n}\left(\alpha^{b}\right)=0 \text { on } \Gamma_{2}, \\
\text { and } \left.\alpha=0 \text { on } \Gamma_{1}\right\},
\end{gathered}
$$

and for $2>r \geq 1$, set

$$
\left.H_{A}^{r}=\left\{\alpha \in H^{r}(\Lambda(M)) \mid n\right\lrcorner \alpha=0 \text { on } \Gamma_{2}, \text { and } \alpha=0 \text { on } \Gamma_{1}\right\} .
$$

Note that the restriction operator to these subspaces is a continuous linear map. $\mathcal{L}$ is a self-adjoint linear unbounded nonnegative operator on $L^{2}$ with $D(\mathcal{L})=H_{A}^{2}$, and $\mathcal{L}: H_{A}^{2} \rightarrow \operatorname{im}(d) \oplus \operatorname{im}(\delta)$ is an isomorphism. It follows that $(1-\mathcal{L}): H_{A}^{2} \rightarrow H^{1}(\Lambda(M))$ is an isomorphism. Since

$$
\mathcal{L}=-(\triangle+2 \operatorname{Ric}+d \delta)
$$

and since we have proven that $\bar{\triangle}_{\eta}, \bar{d}_{\eta}, \bar{\delta}_{\eta}$, and $\overline{\mathrm{Ric}}_{\eta}$ are $C^{\infty}$ bundle maps, it follows that 


$$
\overline{(1-\mathcal{L})}:\left(H_{A}^{r}\right)_{\eta} \downarrow \mathfrak{G}^{s} \rightarrow H_{\eta}^{r-2}(T M) \downarrow \mathfrak{G}^{s}
$$

is a $C^{\infty}$ bundle isomorphism covering the identity, so that by the inverse function theorem,

$$
\overline{(1-\mathcal{L})^{-1}}: H_{\eta}^{r-2}(T M) \downarrow \mathfrak{G}^{s} \rightarrow\left(H_{A}^{r}\right)_{\eta} \downarrow \mathfrak{G}^{s}
$$

is $C^{\infty}$ as well.

This proves the theorem in the case that $\mathfrak{G}^{s}=\mathcal{D}_{\text {mix }}^{s}$. In the case that $\mathfrak{G}^{s}=\mathcal{D}_{N}^{s}$, simply set $\Gamma_{1}=\emptyset$, and for $\mathfrak{G}^{s}=\mathcal{D}_{D}^{s}$, set $\Gamma_{2}=\emptyset$ in the definition of $H_{A}^{r}$. q.e.d.

\section{Acknowledgments}

The author thanks Jerry Marsden, Marcel Oliver, and Tudor Ratiu for many discussions on a variety of topics appearing in this manuscript. Research was partially supported by the NSF-KDI grant ATM-98-73133 and the Alfred P. Sloan Foundation Research Fellowship.

\section{Appendix A. The Euler-Poincaré variational principle}

The reduction of geodesic flow on $\mathcal{D}_{\mu}^{s}$ (or any of its subgroups) onto the single fiber of $T \mathcal{D}_{\mu}^{s}$ over the identity $e$ is an example of the EulerPoincaré theorem (see [22]) which we shall now state in the setting of a topological group $G$ which is a smooth manifold and admits smooth right translation. For any element $\eta$ of the group, we shall denote by $T R_{\eta}$ the right translation map on $T G$, so that for example, when $G$ is either $\mathcal{D}_{\mu, D}^{s}, \mathcal{D}_{\mu, N}^{s}$, or $\mathcal{D}_{\mu, m i x}^{s}$, then $T R_{\eta^{-1}} \dot{\eta}:=\dot{\eta} \circ \eta^{-1}$.

Proposition 6 (Euler-Poincaré). $\quad$ Let $G$ be a topological group which admits smooth manifold structure with smooth right translation, and let $L: T G \rightarrow \mathbb{R}$ be a right invariant Lagrangian. Let $\mathfrak{g}$ denote the fiber $T_{e} G$, and let $l: \mathfrak{g} \rightarrow \mathbb{R}$ be the restriction of $L$ to $\mathfrak{g}$. For a curve $\eta(t)$ in $G$, let $u(t)=T R_{\eta(t)-1} \dot{\eta}(t)$. Then the following are equivalent:

a the curve $\eta(t)$ satisfies the Euler-Lagrange equations on $G$;

$\mathbf{b}$ the curve $\eta(t)$ is an extremum of the action function

$$
S(\eta)=\int L(\eta(t), \dot{\eta}(t)) d t
$$

for variations $\delta \eta$ with fixed endpoints; 
c the curve $u(t)$ solves the Euler-Poincaré equations

$$
\frac{d}{d t} \frac{\delta l}{\delta u}=-a d_{u}^{*} \frac{\delta l}{\delta u}
$$

where the coadjoint action $a d_{u}^{*}$ is defined by

$$
\left\langle a d_{u}^{*} v, w\right\rangle=\left\langle v,[u, w]_{R}\right\rangle
$$

for $u, v, w$ in $\mathfrak{g}$, and where $\langle\cdot, \cdot\rangle$ is the metric on $\mathfrak{g}$ and $[\cdot, \cdot]_{R}$ is the right bracket;

$\mathbf{d}$ the curve $u(t)$ is an extremum of the reduced action function

$$
s(u)=\int l(u(t)) d t
$$

for variations of the form

$$
\delta u=\dot{w}+[w, u]_{R},
$$

where $w=T R_{\eta^{-1}} \delta \eta$ vanishes at the endpoints.

See Chapter 13 in [22] for a detailed development of the theory of Lagrangian reduction as well as a proof of the Euler-Poincaré theorem.

\section{References}

[1] S. Agmon, A. Douglis \& L. Nirenberg, Estimates near the boundary for solutions of elliptic partial differential equations satisfying general boundary conditions. II, Comm. Pure Appl. Math. 17 (1964) 35-72.

[2] R. Abraham, J.E. Marsden \& T. Ratiu, Manifolds, tensor analysis, and applications, Springer, New York, 1988.

[3] V. I. Arnold, Sur la geometrie differentielle des groupes de Lie de dimension infinie et ses applications a l'hydrodynamique des fluids parfaits, Ann. Inst. Grenoble, 16 (1966) 319-361.

[4] V. I. Arnold \& B. Khesin, Topological Methods in Hydrodynamics, Springer, New York, 1998.

[5] G. I. Barenblatt \& A. J. Chorin, New Perspectives in turbulence: scaling laws, asymptotics, and intermittency, SIAM Rev. 40 (1998) 265-291. 
[6] Y. Brenier, Minimal geodesics on groups of volume-preserving maps and generalized solutions to the Euler equations, Comm. Pure Appl. Math. 52 (1999) 411-452.

[7] S. Chen, C. Foias, D. D. Holm, E. Olson, E. S. Titi \& S. Wynne, Camassa-Holm equations as a closure model for turbulent channel and pipe flow, Phys. Rev. Lett. 81 (1998) 5338-5341.

[8] _ A connection between the Camassa-Holm equations and turbulent flows in channels and pipes, The Internat. Conf. Turbulence (Los Alamos, NM, 1998). Phys. Fluids 11 (1999) 2343-2353.

[9] - The Camassa-Holm equations and turbulence, Predictability: quantifying uncertainty in models of complex phenomena (Los Alamos, NM, 1998). Phys. D 133 (1999) 49-65.

[10] S. Chen, D.D. Holm, L.G. Margolin \& R. Zhang, Direct numerical simulations of the Navier-Stokes alpha model, Predictability: quantifying uncertainty in models of complex phenomena, (Los Alamos, NM, 1998). Phys. D, 133 (1999) 66-83.

[11] A. Chorin, Numerical study of slightly viscous flow, J. Fluid Mech. 57 (1973) $785-796$.

[12] G. Duff, Differential forms in manifolds with boundary, Ann. of Math. 56 (1952) $115-127$.

[13] D. Ebin \& J. Marsden, Groups of diffeomorphisms and the motion of an incompressible fluid, Ann. of Math. 92 (1970) 102-163.

[14] J. Eells, A setting for global analysis, Bull. Amer. Math. Soc. 72 (1966) 751-807.

[15] H. Eliasson, Geometry of manifolds of maps, J. Differential Geom. 1 (1967) 169194.

[16] G. Fichera, Existence theorems in elasticity, Encyclopedia of Physics, Springer, (1972) 347-390.

[17] C. Foias, D. D. Holm \& E.S. Titi, The three dimensional viscous Cammassa-Holm Equations and their relation to the Navier-Stokes equations and turbulence theory, J. Dyn. Differential Equations (1999), to appear.

[18] _ The Navier-Stokes- $\alpha$ model of turbulence, Phys. D, (2001), to appear.

[19] D.D. Holm, J.E. Marsden \& T.S. Ratiu, Euler-Poincaré equations and semidirect products with applications to continuum theories, Adv. Math. 137 (1998) 1-81.

[20] _ Euler-Poincaré models of ideal fluids with nonlinear dispersion, Phys. Rev. Lett. 80 (1998) 4273-4277.

[21] S. Lang, Differentiable and Riemannian manifolds, Springer, New York, (1995).

[22] J. E. Marsden \& T. S. Ratiu, Introduction to mechanics and symmetry, Springer, Second Ed., 1999. 
[23] J. E. Marsden, T. S. Ratiu \& S. Shkoller, A nonlinear analysis of the averaged Euler equations and a new diffeomorphism group, Geom. Funct. Anal. 10 (2000) $582-599$.

[24] J. E. Marsden \& S. Shkoller, The anisotropic Lagrangian averaged Euler and Navier-Stokes equations, Arch. Rational Mech. Anal., (2001), to appear, E-print, http://xyz.lanl.gov/abs/math.AP/0005033/.

[25] - Global well-posedness for the LANS equations on bounded domains Phil. Trans. R. Soc. Lond. A, 2001, to appear.

[26] V. G. Maz'ya, B. A. Plamenevskii \& L. I. Stupyalis, The three-dimensional problem of steady-state motion of a fluid with a free surface, Amer. Math. Soc. Tranl. 123 (1984) 171-268.

[27] K. Mohseni, S. Shkoller, B. Kosović, J. E. Marsden, D. Carati, A. Wray \& R. Rogallo, Numerical simulations of homogeneous turbulence using the Lagrangian averaged Navier-Stokes equations, Center for Turbulence Research, Proc. Summer Program, 2000, 271-283.

[28] C. B. Morrey, Multiple integrals in the calculus of variations, Springer, 1966.

[29] B. Nadiga \& S. Shkoller, Enhancement of the inverse-cascade of energy in the two-dimensional Lagrangian-averaged Navier-Stokes equations, Phys. of Fluids 13 (2001) 1528-1531.

[30] M. Oliver \& S. Shkoller, The vortex blob method as a second-grade non-Newtonian fluid, Comm. Partial Differential Equations 26 (2001) 91-110.

[31] R. Palais, Foundations of global nonlinear analysis, Benjamin, New York, 1968.

[32] S. Pekarsky \& S. Shkoller, unpublished notes.

[33] R. S. Rivlin \& J. L. Ericksen, Stress-deformation relations for isotropic materials, J. Rat. Mech. Anal. 4 (1955) 323-425.

[34] S. Shkoller, Geometry and curvature of diffeomorphism groups with $H^{1}$ metric and mean hydrodynamics, J. Funct. Anal. 160 (1998) 337-365.

[35] _ Smooth global Lagrangian flow for the 2D Euler and second-grade fluid equations, Appl. Math. Lett. 14 (2001) 539-543.

[36] A. I. Shnirelman, The geometry of the group of diffeomorphisms and the dynamics of an ideal incompressible fluid, (Russian) Mat. Sb. (N.S.) 128 (1985) 82-109.

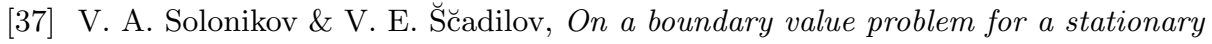
system of Navier-Stokes equations, Proc. Steklov Inst. Math. 125 (1973) 186-199.

[38] M. E. Taylor, Partial differential equations. I, II, III, Springer, 1996.

[39] R. Temam, Infinite-Dimensional Dynamical Systems in Mechanics and Physics, Springer-Verlag, 1988.

\section{University of CALIFORNiA}

